DAMTP-2008-116

arXiv:0901.0450 [hep-th]

\title{
Reparameterisation Invariance and RG equations: Extension of the Local Potential Approximation
}

\author{
H. Osborn ${ }^{1}$ and D.E. Twigg ${ }^{2}$ \\ Department of Applied Mathematics and Theoretical Physics, \\ Wilberforce Road, Cambridge, CB3 0WA, England
}

\begin{abstract}
Equations related to the Polchinski version of the exact renormalisation group equations for scalar fields which extend the local potential approximation to first order in a derivative expansion, and which maintain reparameterisation invariance, are postulated. Reparameterisation invariance ensures that the equations determine the anomalous dimension $\eta$ unambiguously and the equations are such that the result is exact to $\mathrm{O}\left(\varepsilon^{2}\right)$ in an $\varepsilon$-expansion for any multi-critical fixed point. It is also straightforward to determine $\eta$ numerically. When the dimension $d=3$ numerical results for a wide range of critical exponents are obtained in theories with $O(N)$ symmetry, for various $N$ and for a ranges of $\eta$, are obtained within the local potential approximation. The associated $\eta$, which follow from the derivative approximation described here, are found for various $N$. The large $N$ limit of the equations is also analysed. A corresponding discussion is also given in a perturbative RG framework and scaling dimensions for derivative operators are calculated to first order in $\varepsilon$.
\end{abstract}

PACS:11.10.-z, 11.10.Gh, 64.60.Fr, 64.60Ak, 64.60.Kw, 68.35.Rh

Keywords:Exact Renormalisation Group, Derivative Expansion, Reparameterisation Invariance.

\footnotetext{
${ }^{1}$ ho@damtp.cam.ac.uk

22det28@cam.ac.uk
} 


\section{Introduction}

Exact functional renormalisation group flow equations $[1,2,3,4,5,6,7,8]$ allow, at least for scalar field theories, the possibility of a non perturbative analysis of fixed points and determination of critical exponents which control the RG flow near any fixed point. In all such equations there is a cut off function $K\left(p^{2}\right)$ which is essentially arbitrary save for $K(0)=1$ and vanishing sufficiently rapidly as $p^{2} \rightarrow \infty$. Any physical results, such as precise values for exponents, should be independent of the cut off although it may be feasible to optimise over different cut off functions [9]. The exact RG flow equations are hard to handle except in some truncation or expanding in perturbation theory. The local potential approximation (LPA) neglects the spatial dependence of the fields $\phi$ and reduces the effective action $S[\phi]$ from a highly non trivial functional to a simple function $V(\phi)$ and the RG flow equations become a non linear differential equation for $V$ of the form $\dot{V}_{t}=F\left(V_{t}, V_{t}^{\prime}, V_{t}^{\prime \prime}\right)$ where $\dot{V}$ denotes the derivative with respect to $t=-\log \Lambda$, with $\Lambda$ the cut off scale (the equations are invariant under rescalings of $\Lambda$ ). The potential RG flow fixed points, $V_{t} \rightarrow V$ as $t \rightarrow \infty$, are determined by requiring smooth solutions for all $\phi$ of $F\left(V, V^{\prime}, V^{\prime \prime}\right)=0$. The critical exponents describing the $\mathrm{RG}$ flow in the neighbourhood of the fixed point, $V_{t}=V+\sum_{r} \epsilon_{r} e^{\lambda_{r} t} v_{r}$, may then be calculated by finding the eigenvalues $\lambda_{r}$, and eigenfunctions $v_{r}$, for a corresponding linear differential operator depending on the fixed point solution for $V$. For all $\lambda_{r}>0$ the associated operators are relevant and it is necessary to tune $\epsilon_{r}=0$ to attain the fixed point under RG flow.

When applied to the Polchinski RG equation [4], for which $F\left(V, V^{\prime}, V^{\prime \prime}\right)$ has a very simple quadratic form, the LPA has the virtue that all dependence of the cut off can be removed by rescalings of $V$ and $\phi$. Although rather crude the LPA is compatible with the global features of RG flow since, in cases that have been investigated, it realises the same fixed points as are present in the full quantum field theory for scalar fields that are found by other techniques (this is not manifestly true for more complicated theories with gauge fields and fermions [10]).

Despite describing the essential features of the landscape of critical points for scalar theories the LPA has, nevertheless, many limitations. In particular it is not possible to consistently determine $\eta$, the anomalous dimension for $\phi$. In theories with dimension $d=3$, $\eta$ is generally small but clearly results for critical exponents must then have an error of at least $\mathrm{O}(\eta)$, although results when the LPA is applied to different RG flow equations differ in general by rather more than this. Attempts to go beyond the LPA usually invoke an expansion in terms of derivatives of $\phi[11,12,13,14,15,16,17,18]$. To first order this introduces a function $Z(\phi)$ which is the coefficient of $(\partial \phi)^{2}$ in an expansion of the effective action (for multi-component fields $\phi_{i}$ this becomes a symmetric tensor $Z_{i j}(\phi)$ ). $Z$ and $V$ obey coupled equations which in principle allow $\eta$ to be determined by requiring non singular solutions for both $Z$ and $V$. However the dependence on the cut off becomes more severe in the derivative expansion. Applied to the Polchinski equation there are two constants $A, B$ which are essentially arbitrary [13]. Apart from this arbitrariness the results also depend on the value chosen for $Z(0)$ in solving the coupled equations [15].

Exact RG equations, without approximations, are invariant under reparameterisations, including rescalings, of the fundamental fields [19]. This property ensures that the full 
equations have a line of physically equivalent fixed points which may be parameterised by different values of $Z(0),[15,20,5]$. Physical results, such as $\eta$, are independent of where on this line the fixed point solution is chosen. As a consequence of the line of equivalent fixed points the calculated exponents must include one which is exactly zero. The corresponding marginal operator is redundant, essentially one which vanishes on the equations of motion.

In the context of the Polchinski RG equation it was shown, for arbitrary dimensions $d$, in [21] that for any local operator $\mathcal{O}$, such that $\int \mathrm{d}^{d} x \mathcal{O}$ gives an eigen-operator represented by an eigenfunction for the linearised equations with critical exponent $\lambda_{\mathcal{O}}$, then it is possible to construct associated redundant operators with exponents

$$
\lambda=\lambda_{\mathcal{O}}-\frac{1}{2}(d+2-\eta)-2 m, \quad m=0,1,2, \ldots,
$$

irrespective of any particular choice of a smooth cut off function. Furthermore the operator $\phi$ is a local operator determining an eigenfunction with $\lambda_{\phi}=\frac{1}{2}(d+2-\eta)$ and hence, applying (1.1) with $m=0$, this directly shows that $\lambda=0$ is a possible eigenvalue whose eigenfunction generates the marginal operator necessary for reparameterisation invariance.

Although reparameterisation invariance is a property of the full non linear RG equations it is generally lost in approximations such as the derivative expansion. There is no longer a fluctuation eigenfunction with $\lambda=0$ exactly. Here we heuristically construct equations for $V(\phi), Z(\phi)$, and also for multi-component generalisations, which maintain these desirable features. The equation for $V$ remains the same as in the LPA except for the introduction of $\eta$. The associated equation for $Z$ depends on the solution for $V$ and determines $\eta$. Using an appropriate scalar product an integral expression may be found which may be used to find $\eta$ in a fashion which is manifestly independent of $Z(0)$. The eigenvalues for the corresponding differential operator are in accord with (1.1) when $m=0$ and the zero mode eigenfunction can also be found explicitly.

For the purposes of comparison we also discuss results for derivative operators of the form $G_{i j}(\phi) \partial^{\mu} \phi_{i} \partial_{\mu} \phi_{j}$ using standard perturbation theory techniques and the $\varepsilon$-expansion to obtain results for the anomalous dimensions at the fixed point to first order in $\varepsilon$. For such derivative operators it is necessary to take account of mixing with scalar operators $F(\phi)$ with the same dimension but there are also additional constraints on the associated $\beta$-functions. Keeping only contributions just to first order in the coupled $\left(F, G_{i j}\right)$ then an infinitesimal variation $\delta \phi_{i}=v_{i}(\phi)$, for non-linear $v_{i}(\phi)$, in the lagrangian is equivalent to corresponding changes in $\left(F, G_{i j}\right)$. This leads to identities which show that the scaling dimensions satisfy relations of the same form as in (1.1) in general in a perturbative framework. We are also able to determine the scale dimensions to $\mathrm{O}(\varepsilon)$ at each of the multi-critical fixed points for scalar theories when $O(N)$ symmetry is imposed. Although such operators are irrelevant as far as RG flows they are of course of interest in determining the spectrum of operators and scale dimensions in the theory at its critical points.

In this paper we describe in the next section results for the simplest case of a single component field which corresponds to the Ising model and has been much discussed previously. For $d=3$ the equations are solved numerically and the associated eigenvalues determined for various values of $\eta$. The appropriate value of $\eta$ necessary for a non singular solution of the $Z$-equation is also found. In section 3 , we extend the discussion to 
multi-component fields, imposing $O(N)$ symmetry so that simple equations, of similar form to those considered in section 2, are obtained. The eigenfunctions are then $O(N)$ tensors. The irreducible representations are given by symmetric traceless tensors of rank $l$ and the corresponding eigenvalues depend also om $l$. Numerical results are then given for various $N$ and $l$. In section 4 we show how these equations may be solved in an $\varepsilon$-expansion recovering perturbative results at the various possible non-trivial multi-critical fixed points as the dimension $d$ is reduced. In section 5 we consider perturbatively the usual $\beta$-functions in a loop expansion, extending results obtained in the single component case in [21]. In section 6 these results are extended to derivative operators and mixing effects taken into account. In a conclusion we make some more general remarks concerning the status of the equations discussed in this paper. Although they have been motivated by requiring that they share general properties of the exact RG equations they serve to show how these may still be maintained in quite simple approximations. Various calculational details are relegated to four appendices. In appendix A we show how the equations can be solved for large $N$ and a formula for $\eta$ to $\mathrm{O}\left(N^{-1}\right)$ obtained which is quite close to the exact large $N$ result. In appendix $\mathrm{B}$ we give some details of the perturbative results for $\beta$-functions that are used in sections 5 and 6 . In appendix $\mathrm{C}$ we give a general discussion using dimensional regularisation of the consequences of invariance of the regularised theory under variations $\delta \phi_{i}=-v_{i}(\phi)$. In the final appendix $\mathrm{D}$ we give some details of the nearest singularities that are found numerically when the solution of the local potential approximation for the Polchinski equation is extended to the complex plane.

\section{Equations for a Single Component Field}

It is simplest to consider first a single scalar field $\phi$ corresponding to the universality class for the Ising model. At a fixed point the equation for $V(\phi)$ is

$$
V^{\prime \prime}(\phi)-\frac{1}{2}(d-2+\eta) \phi V^{\prime}(\phi)-V^{\prime}(\phi)^{2}+d V(\phi)=0 .
$$

This is just the standard LPA for the Polchinski equation including the anomalous dimension $\eta$. In general this is set to zero as there is no mechanism to determine this from (2.1). The two trivial solutions of $(2.1)$ are $V(\phi)=0$, for the Gaussian fixed point, and $V(\phi)=$ $\frac{1}{4}(2-\eta)\left(\phi^{2}-\frac{2}{d}\right)$, for the high temperature fixed point. Non trivial solutions even in $\phi$, so that $V^{\prime}(0)=0$, which are non singular for all $\phi$ and

$$
V(\phi) \sim \frac{1}{4}(2-\eta) \phi^{2}+A \phi^{\frac{2 d}{d+2-\eta}} \quad \text { for large } \quad \phi,
$$

depend on a precise choice for $V(0)=k$ which then determines $A$. Such solutions appear whenever $d$ is reduced below $2 n /(n-1)$ for $n=2,3, \ldots$ [22, 23]. The critical exponents are then determined from the eigenvalue equation

$$
\mathcal{D} f(\phi)=(\lambda-d) f(\phi),
$$

with the differential operator

$$
\mathcal{D}=\frac{\mathrm{d}^{2}}{\mathrm{~d} \phi^{2}}-\frac{1}{2}(d-2+\eta) \phi \frac{\mathrm{d}}{\mathrm{d} \phi}-2 V^{\prime}(\phi) \frac{\mathrm{d}}{\mathrm{d} \phi} .
$$


It is easy to see, using (2.1), that

$$
\mathcal{D} V^{\prime}(\phi)=-\frac{1}{2}(d+2-\eta) V^{\prime}(\phi), \quad \mathcal{D} \phi=-\frac{1}{2}(d-2+\eta) \phi-2 V^{\prime}(\phi),
$$

and hence we may construct two exact odd eigenfunctions

$$
f_{\phi}(\phi)=\frac{1}{2}(2-\eta) \phi-V^{\prime}(\phi), \quad \lambda_{\phi}=\frac{1}{2}(d+2-\eta), \quad f_{R}(\phi)=V^{\prime}(\phi), \quad \lambda_{R}=\frac{1}{2}(d-2+\eta),
$$

$f_{R}(\phi)$ corresponds to a redundant operator with $\lambda_{R}$ given by (1.1) with $\mathcal{O}$ the identity operator, which corresponds to the solution of $(2.3) f(\phi)=1$ with $\lambda=d$.

It is important for our later discussion to recognise that $\mathcal{D}$ in $(2.4)$ is hermitian with respect to a scalar product defined by

$$
\langle f, g\rangle=\int \mathrm{d} \phi e^{-\frac{1}{4}(d-2+\eta) \phi^{2}-2 V(\phi)} f(\phi) g(\phi),
$$

so that

$$
\langle f, \mathcal{D} g\rangle=\langle\mathcal{D} f, g\rangle
$$

Extending the RG equations to $Z(\phi)$ we propose that, in conjunction with (2.1), the associated equation at the fixed point

$$
\begin{array}{r}
Z^{\prime \prime}(\phi)-\frac{1}{2}(d-2+\eta) \phi Z^{\prime}(\phi)-2 V^{\prime}(\phi) Z^{\prime}(\phi)-2 V^{\prime \prime}(\phi) Z(\phi) \\
=\left(\mathcal{D}-2 V^{\prime \prime}(\phi)\right) Z(\phi)=\eta-\frac{2 d}{d+2} V^{\prime \prime}(\phi)^{2} .
\end{array}
$$

Together with (2.1) this satisfies reparameterisation invariance so that $\eta$ is independent of the particular initial $Z(0)$, unlike the case for other analogous derivative expansion equations. To ensure non singular solutions for all $\phi$ requires only a special choice for $\eta$. Asymptotically, for large $\phi$, solutions of (2.9) have the form

$$
Z(\phi) \sim \frac{d(2-\eta)}{2(d+2)}-\frac{\eta}{2-\eta}+C \phi^{-\frac{2(2-\eta)}{d+2-\eta}}
$$

In general the value of the asymptotic constant $C$ depends on $Z(0)$.

The proposed $Z$-equation (2.9) is similar to the derivative expansion result in [13]. It differs in that the coefficient of the $V^{\prime \prime} Z$ term is 2 , rather than 4 , and that on the right hand side there is a definite coefficient $\frac{2 d}{d+2}$ rather than an essentially arbitrary cut off dependent constant (in terms of the equations in [13] we are taking for the cut dependent constant $B$ the precise value $\left.\frac{d}{d+2}\right)$. In respect of these terms (2.9) is identical with an analogous equation obtained in [21] using an expansion in terms of scaling fields which is similar in spirit to the derivative expansion. In the scaling field approach the corresponding coefficient is determined precisely essentially by those divergencies in two point amplitudes which are universal, i.e. renormalisation scheme independent. If $G(x)$ is the cut off dependent propagator, then we have for the following products

$$
\frac{\partial}{\partial t} G(x)^{n} \sim-n a_{n} \delta^{d}(x), \quad \frac{\partial}{\partial t} G(x)^{2 n-1} \sim-(2 n-1) b_{n} \partial^{2} \delta^{d}(x),
$$


for

$$
d=d_{n}=\frac{2 n}{n-1},
$$

such that $a_{n}, b_{n}$ are constants, independent of the cut off function and depending only on the large $x$ behaviour of $G(x)$. According to the results obtained in [21]

$$
\frac{b_{n}}{a_{n}^{2}}=\frac{d_{n}}{2\left(d_{n}+2\right)} \text {. }
$$

The coefficients in RG equations such as (2.9) should not depend on the particular critical point, here labelled by $n$, but may depend on the spatial dimension $d$. Applying the $\varepsilon$ expansion to (2.9) with the particular coefficient $\frac{2 d}{d+2}$ ensures, as was shown in [21] and also subsequently here, that $\eta$ is correct to $\mathrm{O}\left(\varepsilon^{2}\right)$ for all critical points $n=2,3, \ldots$ Although results such as these for $\eta$ were obtained from Wilsonian RG equations as soon as they were first proposed, and were shown to be independent of the detailed cut off function [24], they are also identical, of course, with results from standard Feynman graph techniques which arise directly from the coefficients of the universal logarithmic divergencies for particular two point Feynman graphs. These logarithmic divergencies are equivalent to (2.11). In a sense compatibility with the $\varepsilon$-expansion may be regarded as an optimal choice for such constants as $B$. However, in the scaling field derivation described in [21] there is no free constant to determine and agreement with the $\varepsilon$-expansion is not imposed but follows automatically.

In addition (2.9) differs from corresponding equations in [13] and [21] by the absence of a $\eta Z$ term. Removing such a contribution is essential to obtain subsequent results. In general in a derivative expansion there are also expected to be additional contributions on the right hand side of (2.1) involving $Z$ but the exact form differs between [13] and [21] and also involves a cut off dependent constant which we are here essentially setting to zero.

Corresponding to (2.1) and (2.9) there are associated eigenvalue equations for critical exponents

$$
\left(\begin{array}{cc}
\mathcal{D}+d & 0 \\
-2 Z^{\prime}(\phi) \frac{\mathrm{d}}{\mathrm{d} \phi}-\left(2 Z(\phi)-\frac{4 d}{d+2} V^{\prime \prime}(\phi)\right) \frac{\mathrm{d}^{2}}{\mathrm{~d} \phi^{2}} & \mathcal{D}-2 V^{\prime \prime}(\phi)
\end{array}\right)\left(\begin{array}{c}
f(\phi) \\
g(\phi)
\end{array}\right)=\lambda\left(\begin{array}{c}
f(\phi) \\
g(\phi)
\end{array}\right) .
$$

It is easy to see that this decouples into pairs of equations for eigenvalues $\lambda_{f}, \lambda_{g}$ where $\lambda_{f}$ is obtained from (2.3), with the corresponding $g$ determined in terms of $f$ by inverting $\mathcal{D}-2 V^{\prime \prime}(\phi)-\lambda_{f}$, and also, with $f=0$,

$$
\mathcal{D} g(\phi)-2 V^{\prime \prime}(\phi) g(\phi)=\lambda_{g} g(\phi) .
$$

For the eigenfunctions in (2.6) the corresponding functions $g$ are given by

$$
-g_{\phi}(\phi)=g_{R}(\phi)=Z^{\prime}(\phi) .
$$

To show reparameterisation invariance of (2.9) we note that for any solution of $(2.3)$ there is a corresponding solution of (2.15) given by

$$
g(\phi)=f^{\prime}(\phi), \quad \lambda_{g}=\lambda_{f}-\frac{1}{2}(d+2-\eta),
$$


in accord with (1.1). Starting from $f_{\phi}(\phi)$ in (2.6) it is then easy to obtain an exact zero mode

$$
g_{0}(\phi)=\frac{1}{2}(2-\eta)-V^{\prime \prime}(\phi),
$$

representing the necessary marginal redundant operator present in the RG flow equations. Since $\mathcal{D}-2 V^{\prime \prime}(\phi)$ is hermitian with respect to the scalar product in $(2.7)$ we must have, for consistent solutions of $(2.9)$,

$$
\begin{aligned}
\eta\left\langle g_{0}, 1\right\rangle & =\eta \int \mathrm{d} \phi e^{-\frac{1}{4}(d-2+\eta) \phi^{2}-2 V(\phi)}\left(\frac{1}{2}(2-\eta)-V^{\prime \prime}(\phi)\right) \\
& =\frac{2 d}{d+2}\left\langle g_{0}, V^{\prime \prime 2}\right\rangle+\left\langle g_{0},\left(\mathcal{D}-2 V^{\prime \prime}\right) Z\right\rangle \\
& =\frac{2 d}{d+2} \int \mathrm{d} \phi e^{-\frac{1}{4}(d-2+\eta) \phi^{2}-2 V(\phi)}\left(\frac{1}{2}(2-\eta)-V^{\prime \prime}(\phi)\right) V^{\prime \prime}(\phi)^{2}
\end{aligned}
$$

Since $\eta$ is small it is easy to iterate (2.19) in conjunction with (2.1) starting from $\eta=0$ to determine the consistent solution for $\eta$ with high numerical precision.

When $d=3$ we may readily solve (2.1) numerically tuning $k=V(0)$ so that the singularity in the solution arises for the largest possible value of $\phi$ compatible with numerical precision, (2.1) was written as two coupled first order equations and were integrated from $\phi=0$ using RK4. The limiting results when $\eta=0$ are shown in Figure 1. Numerical re-

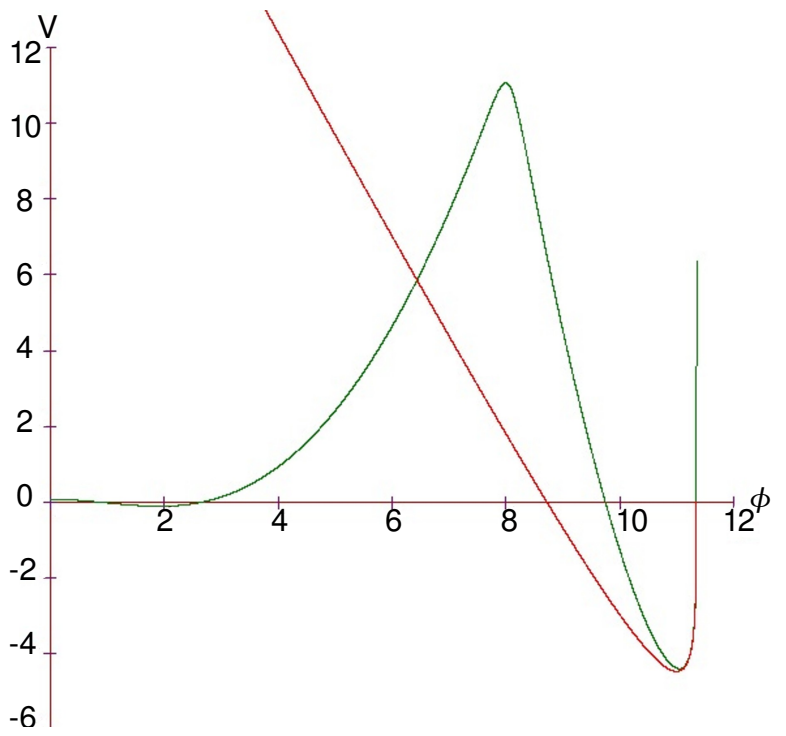

Figure 1: Typical numerical solution of (2.1), with $d=3, \eta=0$, for $V(\phi)$ starting from the critical value $k=V(0)=0.0761994008$. Due to rounding errors the solution breaks down for $\phi \gtrsim 7$ and is singular at $\phi_{0} \approx 11.23$. As shown, for $\phi \lesssim \phi_{0}$, it is well approximated near the singularity by the leading singular form $V_{\text {sing. }}(\phi)=-\ln \left(\phi_{0}-\phi\right)+\frac{1}{4} \phi_{0}\left(\phi_{0}-\phi\right)+$ const. for solutions of (2.1). Note that this has a minimum at $\phi=\phi_{0}-4 / \phi_{0}$ matching the minimum of the numerical solution.

sults for the $Z$-equation (2.9) are also shown in Figure 2. These were obtained in a similar 
fashion as for $V$ in terms of corresponding first order differential equations. The solutions also develop singularities which are very sensitive to the value of $\eta$, where the corresponding $V$-solution of course has been used.

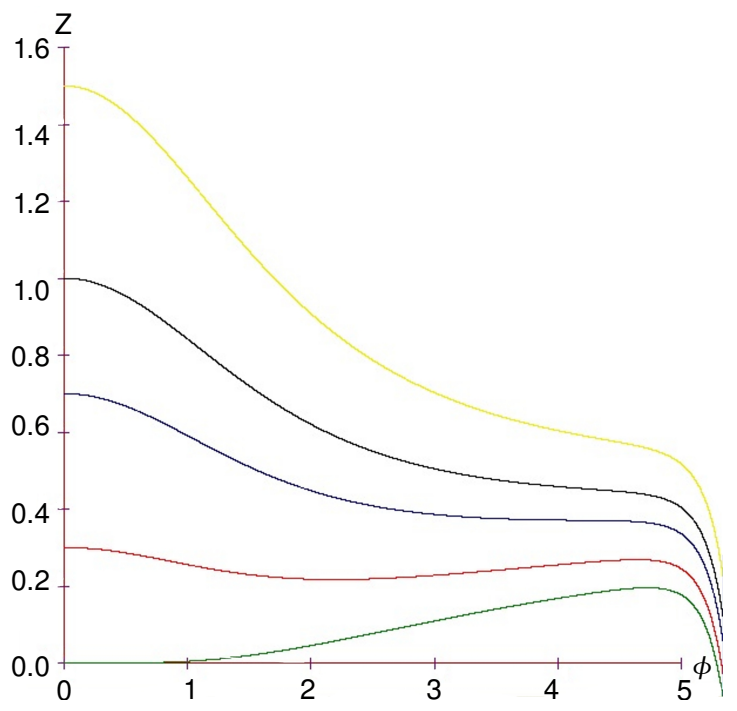

(a) eta $=0.041346$

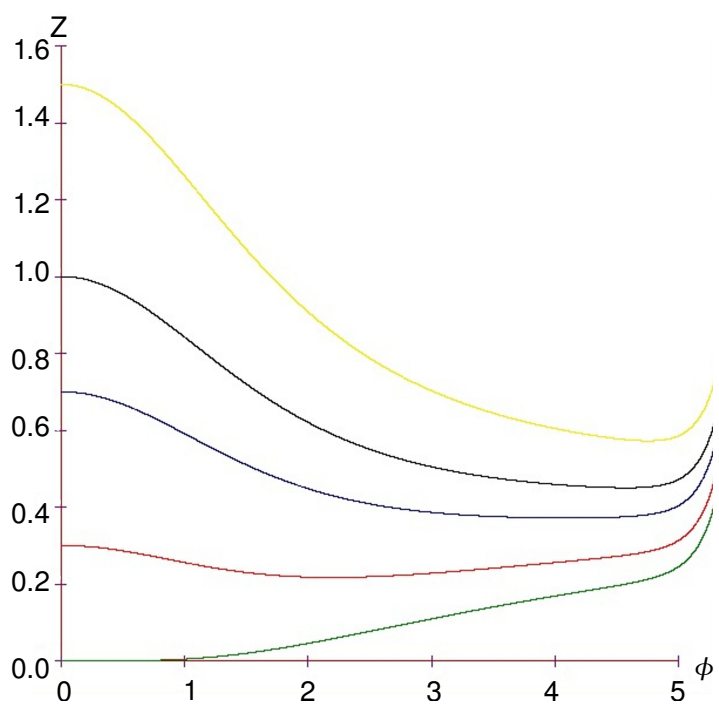

(b) eta $=0.041347$

Figure 2: Numerical solutions of (2.9) for various $Z(0)$ with $\eta$ just either side of the critical value so that the singularity arises for the largest possible $\phi$. The graphs demonstrate how $\eta$ is independent of $Z(0)$.

Having determined $V$ the eigenvalues $\lambda_{n}$ are then determined numerically for small values of $\eta$ and $d=3$ by optimising the eigenvalue such that the eigenfunction blows up as slowly as possible withing the range where results for $V(\phi)$ are reliable. The results are ordered such that $\lambda_{n}>\lambda_{n+1}$ with $\lambda_{0}=3$. For $n$ even,odd the associated eigenfunctions are 
even,odd in $\phi$. From (2.6) $\lambda_{1}=\frac{1}{2}(5-\eta)$ and $\lambda_{3}=\frac{1}{2}(1+\eta)$ which provides a consistency check on our numerical results. For even $n$ the results are in Table 1 and for odd $n$ in Table 2. For the small values considered the dependence on $\eta$ is close to linear. For $\eta=0$ our results agree with the much more accurate determinations in $[25,26]$.

\begin{tabular}{|c|c|c|c|c|c|c|}
\hline$\eta$ & $k$ & $\lambda_{2}$ & $\lambda_{4}$ & $\lambda_{6}$ & $\lambda_{8}$ & $\lambda_{10}$ \\
\hline 0.00 & 0.076199401 & 1.5395 & -0.6557 & -3.1800 & -5.9122 & -8.7961 \\
0.01 & 0.073512228 & 1.5409 & -0.6449 & -3.1560 & -5.8735 & -8.7416 \\
0.02 & 0.070885912 & 1.5421 & -0.6341 & -3.1319 & -5.8344 & -8.6866 \\
0.03 & 0.068319137 & 1.5433 & -0.6232 & -3.1076 & -5.7951 & -8.6312 \\
0.04 & 0.065810847 & 1.5444 & -0.6123 & -3.0832 & -5.7554 & -8.5754 \\
0.05 & 0.063359963 & 1.5454 & -0.6013 & -3.0585 & -5.7155 & -8.5190 \\
0.06 & 0.060965439 & 1.5463 & -0.5903 & -3.0337 & -5.6751 & -8.4621 \\
0.07 & 0.058626258 & 1.5471 & -0.5793 & -3.0087 & -5.6345 & -8.4048 \\
0.08 & 0.056341435 & 1.5478 & -0.5682 & -2.9835 & -5.5935 & -8.3469 \\
0.09 & 0.054110012 & 1.5485 & -0.5570 & -2.9582 & -5.5521 & -8.2884 \\
0.10 & 0.051931059 & 1.5490 & -0.5458 & -2.9326 & -5.5103 & -8.2294 \\
\hline
\end{tabular}

Table 1: Even Eigenvalues and initial value $k=V(0)$ for non singular solutions

\begin{tabular}{|c|c|c|c|}
\hline$\eta$ & $\lambda_{5}$ & $\lambda_{7}$ & $\lambda_{9}$ \\
\hline 0.00 & -1.8867 & -4.5244 & -7.3377 \\
0.01 & -1.8696 & -4.4932 & -7.2911 \\
0.02 & -1.8524 & -4.4618 & -7.2422 \\
0.03 & -1.8351 & -4.4301 & -7.1970 \\
0.04 & -1.8177 & -4.3982 & -7.1493 \\
0.05 & -1.8002 & -4.3660 & -7.1012 \\
0.06 & -1.7826 & -4.3336 & -7.0527 \\
0.07 & -1.7648 & -4.3010 & -7.0038 \\
0.08 & -1.7470 & -4.2680 & -6.9545 \\
0.09 & -1.7290 & -4.2348 & -6.9047 \\
0.10 & -1.7109 & -4.2013 & -6.8544 \\
\hline
\end{tabular}

Table 2: Odd Eigenvalues

We have also determined the value of $\eta$ required for non singular solutions of (2.9) and verified that the result is independent of the value chosen for $Z(0)$ and also in agreement with (2.19). This may be used to determine $\eta$ by iteration starting from $\eta=0$ and gives

$$
\eta=0.041347 \quad \text { for } \quad k=0.0654776 \text {. }
$$

For this value

$$
\nu=1 / \lambda_{2}=0.647, \quad \omega=-\lambda_{4}=0.612 .
$$




\section{Multi-Component Fields}

There are natural generalisations of the above equations to the case of a $N$-component scalar field $\phi_{i}$. Instead of (2.1) we have

$$
\partial^{2} V(\phi)-\frac{1}{2}((d-2) \phi+(\eta \phi)) \cdot \frac{\partial}{\partial \phi} V(\phi)-\frac{\partial}{\partial \phi} V(\phi) \cdot \frac{\partial}{\partial \phi} V(\phi)+d V(\phi)=0,
$$

for $\eta_{i j}$ a symmetric anomalous dimension matrix and $\partial^{2}=\frac{\partial}{\partial \phi} \cdot \frac{\partial}{\partial \phi}$. The corresponding equation for critical exponents is just as in (2.3),

$$
\mathcal{D} F(\phi)=(\lambda-d) F(\phi),
$$

but with the differential operator

$$
\mathcal{D}=\partial^{2}-\frac{1}{2}((d-2) \phi+(\eta \phi)) \cdot \frac{\partial}{\partial \phi}-2 \frac{\partial}{\partial \phi} V(\phi) \cdot \frac{\partial}{\partial \phi},
$$

and we may now also allow tensorial eigenfunctions $F_{i j \ldots}(\phi)$. Trivially $\mathcal{D} 1=0$ so that $\lambda=d$ is an exact eigenvalue. Just as in (2.6) we have exact vector eigenfunctions since

$$
\mathcal{D} F_{\phi, i}(\phi)=\frac{1}{2}(d-2) F_{\phi, i}(\phi)+\frac{1}{2}\left(\eta F_{\phi}(\phi)\right)_{i}, \quad F_{\phi, i}(\phi)=\phi_{i}-\frac{1}{2}(\eta \phi)_{i}-\frac{\partial}{\partial \phi_{i}} V(\phi),
$$

and we may choose a diagonal basis for $\eta_{i j}$. The corresponding scalar product to (2.7) is just

$$
\langle F, G\rangle=\int \mathrm{d}^{N} \phi e^{-\frac{1}{4}(d-2) \phi^{2}-\frac{1}{4} \phi \cdot(\eta \phi)-2 V(\phi)} F(\phi) G(\phi),
$$

with additional tensorial contractions if required.

Extending (2.9) to $Z_{i j}(\phi)$ we require

$$
\mathcal{D} Z_{i j}(\phi)-2 V_{(i k}(\phi) Z_{j) k}(\phi)=\eta_{i j}-\frac{2 d}{d+2} V_{i k}(\phi) V_{j k}(\phi), \quad V_{i j}(\phi)=\frac{\partial^{2}}{\partial \phi_{i} \partial \phi_{j}} V(\phi),
$$

with ( ) used to denote symmetrisation of the $i, j$ indices. For fluctuations $F, G_{i j}$ around the fixed point solutions we have (3.2) and also the associated coupled equation

$$
\begin{aligned}
& \mathcal{D} G_{i j}(\phi)-2 V_{(i k}(\phi) G_{j) k}(\phi) \\
& -2 \partial_{k} Z_{i j}(\phi) \partial_{k} F(\phi)-\left(2 Z_{(i k}(\phi)-\frac{4 d}{d+2} V_{(i k}(\phi)\right) \partial_{j)} \partial_{k} F(\phi)=\lambda G_{i j}(\phi) .
\end{aligned}
$$

As before it is easy to see that the possible eigenvalues are $\lambda_{F}$ determined by (3.2) and $\lambda_{G}$ given by

$$
\mathcal{D} G_{i j}(\phi)-2 V_{(i k}(\phi) G_{j) k}(\phi)=\lambda_{G} G_{i j}(\phi),
$$

corresponding to (2.15). For any vector eigenfunction $F_{i}(\phi)$ there are associated eigenfunctions for (3.8) given in terms of

$$
G_{i j}(\phi)=\partial_{i} F_{j}(\phi)+\partial_{j} F_{i}(\phi),
$$


since

$$
\mathcal{D} G_{i j}(\phi)-2 V_{(i k}(\phi) G_{j) k}(\phi)=\left(\lambda_{F}-\frac{1}{2}(d-2)\right) G_{i j}(\phi)-\frac{1}{2} \eta_{(i k} G_{j) k}(\phi),
$$

Hence we have an exact zero mode which may be obtained from (3.4)

$$
G_{0, i j}(\phi)=\delta_{i j}-\frac{1}{2} \eta_{i j}-V_{i j}(\phi) .
$$

In consequence for (3.6) to be solvable we must have

$$
\int \mathrm{d}^{N} \phi e^{-\frac{1}{4}(d-2) \phi^{2}-\frac{1}{4} \phi \cdot(\eta \phi)-2 V(\phi)}\left(\delta_{i j}-\frac{1}{2} \eta_{i j}-V_{i j}(\phi)\right)\left(\eta_{i j}-\frac{2 d}{d+2} V_{i k}(\phi) V_{j k}(\phi)\right)=0 .
$$

In order to obtain tractable equations we impose $O(N)$ symmetry so that we need only deal with functions of $\rho=\frac{1}{2} \phi^{2}$ and in this case we must have $\eta_{i j}=\eta \delta_{i j}$. Writing $V(\phi)=v(\rho)$ (3.1) becomes

$$
2 \rho v^{\prime \prime}(\rho)-(d-2+\eta) \rho v^{\prime}(\rho)=-N v^{\prime}(\rho)+2 \rho v^{\prime}(\rho)^{2}-d v(\rho) .
$$

At the origin we must have $N v^{\prime}(0)+d v(0)=0$ and asymptotically

$$
v(\rho) \sim \frac{1}{2}(2-\eta) \rho+A \rho^{\frac{d}{d+2-\eta}} .
$$

To ensure non singular solutions as before it is necessary to fine tune $v(0)$. For critical exponents we consider, if $N>1$, spherical harmonics which are expressible in terms of symmetric traceless tensors or equivalently

$$
F_{l}(\phi)=(t \cdot \phi)^{l} f_{l}(\rho), \quad t^{2}=0, \quad l=0,1,2, \ldots .
$$

The eigenvalue equation (3.2) becomes

$$
\mathcal{D}_{l} f_{l}(\rho)=\left(\lambda_{l}+l \frac{1}{2}(d-2+\eta)-d\right) f_{l}(\rho),
$$

for

$$
\mathcal{D}_{l}=2 \rho \frac{\mathrm{d}^{2}}{\mathrm{~d} \rho^{2}}-(d-2+\eta) \rho \frac{\mathrm{d}}{\mathrm{d} \rho}+(N+2 l) \frac{\mathrm{d}}{\mathrm{d} \rho}-4 \rho v^{\prime}(\rho) \frac{\mathrm{d}}{\mathrm{d} \rho}-2 l v^{\prime}(\rho) .
$$

The relevant boundary conditions are that $f_{l}(\rho)$ is analytic for $\rho \approx 0$ and non singular for $\rho>0$ and $\propto \rho^{\frac{d-\lambda_{l}}{d+2-\eta}-\frac{1}{2} l}$ as $\rho \rightarrow \infty$. Corresponding to (3.4)

$$
f_{1, \phi}(\rho)=\frac{1}{2}(2-\eta)-v^{\prime}(\rho)
$$

satisfies

$$
\mathcal{D}_{1} f_{1, \phi}(\rho)=0,
$$

so that $\lambda_{1, \phi}=\frac{1}{2}(d+2-\eta)$. The scalar product, with respect to which $\mathcal{D}_{l}$ is hermitian, becomes from $(3.5)$

$$
\left\langle f_{l}, g_{l}\right\rangle_{l}=\int_{0}^{\infty} \mathrm{d} \rho \rho^{\frac{1}{2} N+l-1} e^{-\frac{1}{2}(d-2+\eta) \rho-2 v(\rho)} f_{l}(\rho) g_{l}(\rho),
$$


When $N=1$ only $l=0,1$ are relevant, corresponding to even,odd eigenfunctions. In terms of $v(\rho)$, which solves (3.13), (3.12) becomes

$$
\begin{aligned}
& \eta \int_{0}^{\infty} \mathrm{d} \rho \rho^{\frac{1}{2} N-1} e^{-\frac{1}{2}(d-2+\eta) \rho-2 v(\rho)}\left(\frac{1}{2}(2-\eta) N-N v^{\prime}(\rho)-2 \rho v^{\prime \prime}(\rho)\right) \\
& =\frac{2 d}{d+2} \int_{0}^{\infty} \mathrm{d} \rho \rho^{\frac{1}{2} N-1} e^{-\frac{1}{2}(d-2+\eta) \rho-2 v(\rho)} \\
& \quad \times\left(\frac{1}{2}(2-\eta)\left(N v^{\prime}(\rho)^{2}+4 \rho v^{\prime}(\rho) v^{\prime \prime}(\rho)+4 \rho^{2} v^{\prime \prime}(\rho)^{2}\right)\right. \\
& \left.\quad-N v^{\prime}(\rho)^{3}-6 \rho v^{\prime}(\rho)^{2} v^{\prime \prime}(\rho)-12 \rho^{2} v^{\prime}(\rho) v^{\prime \prime}(\rho)^{2}-8 \rho^{3} v^{\prime \prime}(\rho)^{3}\right) .
\end{aligned}
$$

When $N=1$ it is easy to see that this is identical with $(2.19)$ since then $V^{\prime \prime}(\phi)=v^{\prime}(\rho)+$ $2 \rho v^{\prime}(\rho)$.

To decompose (3.6) we write

$$
Z_{i j}(\phi)=\delta_{i j} z(\rho)+\phi_{i} \phi_{j}\left(z^{\prime}(\rho)+y(\rho)\right)=\partial_{(i}\left(\phi_{j} z(\rho)\right)+\phi_{i} \phi_{j} y(\rho),
$$

and using

$$
\begin{aligned}
\mathcal{D} Z_{i j}(\phi)-2 V_{(i k}(\phi) Z_{j) k}(\phi)= & \delta_{i j}\left(\mathcal{D}_{1} z(\rho)+2 y(\rho)\right)+\phi_{i} \phi_{j} \frac{\mathrm{d}}{\mathrm{d} \rho}\left(\mathcal{D}_{1} z(\rho)+2 y(\rho)\right) \\
& +\phi_{i} \phi_{j}\left(\mathcal{D}_{1} y(\rho)-\left(d-2+\eta+4 v^{\prime}(\rho)+4 \rho v^{\prime \prime}(\rho)\right) y(\rho)\right),
\end{aligned}
$$

we may reduce (3.6) to

$$
\mathcal{D}_{1} z(\rho)=\eta-2 y(\rho)-\frac{2 d}{d+2} v^{\prime}(\rho)^{2}
$$

and

$$
\mathcal{D}_{1} y(\rho)-\left(d-2+\eta+4 \rho v^{\prime \prime}(\rho)+4 v^{\prime}(\rho)\right) y(\rho)=-\frac{4 d}{d+2} \rho v^{\prime \prime}(\rho)^{2} .
$$

The equation for $y$ thus decouples from that for $z$ so that (3.25) may be solved and the result used in (3.24) to then determine $z$. Asymptotically $z(\rho)$ approaches a constant, just as in (2.10), but $y(\rho)$ vanishes. Since, as shown in (3.19), $\mathcal{D}_{1}$ has a zero mode $f_{1, \phi}$, given by (3.18), $\eta$ must be fixed to allow a non singular solution by,

$$
\eta\left\langle f_{1, \phi}, 1\right\rangle_{1}=2\left\langle f_{1, \phi}, y\right\rangle_{1}+\frac{2 d}{d+2}\left\langle f_{1, \phi}, v^{\prime 2}\right\rangle_{1}
$$

Using

$$
\begin{aligned}
\int_{0}^{\infty} \mathrm{d} \rho \rho^{\frac{1}{2} N-1} & e^{-\frac{1}{2}(d-2+\eta) \rho-2 v(\rho)} G_{0, i j}(\phi) \phi_{i} \phi_{j} \\
& \times\left(\mathcal{D}_{1} y(\rho)-\left(d-2+\eta+4 \rho v^{\prime \prime}(\rho)+4 v^{\prime}(\rho)\right) y(\rho)\right) \\
& =-2(d-2+\eta)\left\langle f_{1, \phi}, y\right\rangle_{1}
\end{aligned}
$$

we may also obtain

$$
(d-2+\eta)\left\langle f_{1, \phi}, y\right\rangle_{1}=\frac{4 d}{d+2} \int_{0}^{\infty} \mathrm{d} \rho \rho^{\frac{1}{2} N+1} e^{-\frac{1}{2}(d-2+\eta) \rho-2 v(\rho)}\left(\frac{1}{2}(2-\eta)-v^{\prime}(\rho)-2 \rho v^{\prime \prime}(\rho)\right) v^{\prime \prime}(\rho)^{2} .
$$


Combining (3.26) and (3.28) is equivalent to (3.21). If we restrict to scalar fluctuations, without any harmonics, then we may decompose $G_{i j}(\phi)$ in terms of $g(\rho), h(\rho)$ in a similar fashion to (3.22) so that (3.8) reduces to

$$
\mathcal{D}_{1} g(\rho)=\lambda g(\rho),
$$

with $h=0$ and also

$$
\mathcal{D}_{1} h(\rho)-4\left(\rho v^{\prime \prime}(\rho)+v^{\prime}(\rho)\right) h(\rho)=(\lambda+d-2+\eta) h(\rho),
$$

when $\left(\mathcal{D}_{1}-\lambda\right) g(\rho)=-2 h(\rho)$. Manifestly the eigensolutions for $g$ in (3.29) are identical with the solutions of (3.16) for $l=1$. The eigenvalues are related as expected from (1.1) so the solutions of (3.29) represent redundant operators. The eigenvalues determined by the $h$-equation (3.30) give exponents corresponding to genuine physical scaling operators.

Numerically (3.13) and (3.16) can be solved straightforwardly for $d=3$, as before after precisely tuning $k^{(N)}=v(0)$ to ensure non singular solutions for all $\rho>0$. The eigenvalues are denoted by $\lambda_{l, m}^{(N)}$ where $\lambda_{0,0}^{(N)}=3$ and we take $m=0,1,2, \ldots$ In terms of the single component results $\lambda_{0, m}^{(1)}=\lambda_{2 m}$ and $\lambda_{1, m}^{(1)}=\lambda_{2 m+1}$. When $l=0$ some results are given in table 3 , for $N=1$ they match as expected the results given earlier. For $\eta=0$ these agree with results in $[27,28]$. In the large $N$ limit, taking $\eta=0$, then $\lambda_{l, m}^{(N)}=3-2 m-\frac{1}{2} l$.

\begin{tabular}{|c|c|c|c|c|c|c|c|l|}
\hline$\eta$ & $k^{(1)}$ & $\lambda_{0,1}^{(1)}$ & $k^{(2)}$ & $\lambda_{0,1}^{(2)}$ & $k^{(4)}$ & $\lambda_{0,1}^{(4)}$ & $k^{(10)}$ & $\lambda_{0,1}^{(10)}$ \\
\hline 0.00 & 0.076200 & 1.5395 & 0.19934 & 1.4120 & 0.53876 & 1.2432 & 1.7637 & 1.0886 \\
0.01 & 0.073513 & 1.5409 & 0.19176 & 1.4175 & 0.51693 & 1.2535 & 1.6929 & 1.0999 \\
0.02 & 0.070886 & 1.5421 & 0.18439 & 1.4229 & 0.49578 & 1.2634 & 1.6242 & 1.1112 \\
0.03 & 0.068319 & 1.5433 & 0.17723 & 1.4281 & 0.47534 & 1.2732 & 1.5577 & 1.1224 \\
0.04 & 0.065811 & 1.5444 & 0.17026 & 1.4330 & 0.45552 & 1.2823 & 1.4931 & 1.1336 \\
0.05 & 0.063360 & 1.5454 & 0.16349 & 1.4378 & 0.43633 & 1.2921 & 1.4306 & 1.1447 \\
\hline
\end{tabular}

Table 3: First $l=0$ Eigenvalues for $N=1,2,4,10$

\begin{tabular}{|c|c|c|c|c|c|c|c|c|}
\hline$\eta$ & $\lambda_{0,2}^{(1)}$ & $\lambda_{0,2}^{(2)}$ & $\lambda_{0,2}^{(4)}$ & $\lambda_{0,2}^{(10)}$ & $\lambda_{0,3}^{(1)}$ & $\lambda_{0,3}^{(2)}$ & $\lambda_{0,3}^{(4)}$ & $\lambda_{0,3}^{(10)}$ \\
\hline 0.00 & -0.6557 & -0.6712 & -0.7338 & -0.8713 & -3.1800 & -3.0714 & -2.9400 & -2.8985 \\
0.01 & -0.6449 & -0.6596 & -0.7187 & -0.8515 & -3.1560 & -3.0498 & -2.9199 & -2.8728 \\
0.02 & -0.6341 & -0.6480 & -0.7039 & -0.8319 & -3.1319 & -3.0281 & -2.8998 & -2.8473 \\
0.03 & -0.6232 & -0.6364 & -0.6892 & -0.8124 & -3.1076 & -3.0062 & -2.8795 & -2.8221 \\
0.04 & -0.6123 & -0.6247 & -0.6746 & -0.7930 & -3.0832 & -2.9841 & -2.8592 & -2.7970 \\
0.05 & -0.6013 & -0.6131 & -0.6602 & -0.7739 & -3.0585 & -2.9617 & -2.8397 & -2.7722 \\
\hline
\end{tabular}

Table 4: Second and Third $l=0$ Eigenvalues for $N=1,2,4,10$

For $l=1$ then $\lambda_{1,0}^{(N)}=\frac{1}{2}(5-\eta)$ and $\lambda_{1,1}^{(N)}=\frac{1}{2}(1+\eta)$ which is a useful check. Some other results are given in table 5 . We also present some results for $l=2$ in table 6 and $l=4$ in table 7. An important observation is that $\lambda_{4,0}^{(2)}<0$ whereas $\lambda_{4,0}^{(N)}>0$ for $N \geq 3$. This reflects the instability of the $O(N)$ symmetric fixed point against RG flow to one with just 


\begin{tabular}{|c|c|c|c|c|c|c|c|c|}
\hline$\eta$ & $\lambda_{1,2}^{(1)}$ & $\lambda_{1,2}^{(2)}$ & $\lambda_{1,2}^{(4)}$ & $\lambda_{1,2}^{(10)}$ & $\lambda_{1,3}^{(1)}$ & $\lambda_{1,3}^{(2)}$ & $\lambda_{1,3}^{(4)}$ & $\lambda_{1,3}^{(10)}$ \\
\hline 0.00 & -1.8867 & -1.7986 & -1.6741 & -1.5535 & -4.5244 & -4.3251 & -4.0185 & -3.6719 \\
0.01 & -1.8696 & -1.7835 & -1.6615 & -1.5406 & -4.4932 & -4.2984 & -3.9983 & -3.6529 \\
0.02 & -1.8524 & -1.7683 & -1.6487 & -1.5277 & -4.4618 & -4.2714 & -3.9778 & -3.6339 \\
0.03 & -1.8351 & -1.7530 & -1.6358 & -1.5148 & -4.4301 & -4.2442 & -3.9570 & -3.6149 \\
0.04 & -1.8177 & -1.7375 & -1.6228 & -1.5020 & -4.3982 & -4.2166 & -3.9358 & -3.5958 \\
0.05 & -1.8002 & -1.7220 & -1.6096 & -1.4891 & -4.3661 & -4.1887 & -3.9143 & -3.5767 \\
\hline
\end{tabular}

Table $5: l=1$ Eigenvalues for $N=1,2,4,10$

\begin{tabular}{|c|l|l|l|c|c|c|}
\hline$\eta$ & $\lambda_{2,0}^{(2)}$ & $\lambda_{2,0}^{(4)}$ & $\lambda_{2,0}^{(10)}$ & $\lambda_{2,1}^{(2)}$ & $\lambda_{2,1}^{(4)}$ & $\lambda_{2,1}^{(10)}$ \\
\hline 0.00 & 1.7819 & 1.8476 & 1.9283 & -0.4737 & -0.3332 & -0.1531 \\
0.01 & 1.7760 & 1.8396 & 1.9187 & -0.4675 & -0.3308 & -0.1535 \\
0.02 & 1.7701 & 1.8317 & 1.9091 & -0.4612 & -0.3282 & -0.1539 \\
0.03 & 1.7642 & 1.8239 & 1.8996 & -0.4548 & -0.3255 & -0.1541 \\
0.04 & 1.7583 & 1.8161 & 1.8901 & -0.4482 & -0.3226 & -0.1542 \\
0.05 & 1.7525 & 1.8084 & 1.8807 & -0.4416 & -0.3195 & -0.1543 \\
\hline
\end{tabular}

Table $6: l=2$ Eigenvalues for $N=2,4,10$

\begin{tabular}{|c|c|c|c|c|c|c|c|c|}
\hline$\eta$ & $\lambda_{4,0}^{(2)}$ & $\lambda_{4,0}^{(3)}$ & $\lambda_{4,0}^{(4)}$ & $\lambda_{4,0}^{(10)}$ & $\lambda_{4,1}^{(2)}$ & $\lambda_{4,1}^{(3)}$ & $\lambda_{4,1}^{(4)}$ & $\lambda_{4,1}^{(10)}$ \\
\hline 0.00 & -0.0337 & 0.1109 & 0.2315 & 0.6045 & -2.6147 & -2.3954 & -2.2093 & -1.6169 \\
0.01 & -0.0358 & 0.1046 & 0.2218 & 0.5871 & -2.6023 & -2.3889 & -2.2077 & -1.6286 \\
0.02 & -0.0377 & 0.0985 & 0.2124 & 0.5700 & -2.5897 & -2.3821 & -2.2056 & -1.6373 \\
0.03 & -0.0395 & 0.0926 & 0.2032 & 0.5530 & -2.5768 & -2.3749 & -2.2031 & -1.6456 \\
0.04 & -0.0412 & 0.0869 & 0.1943 & 0.5326 & -2.5636 & -2.3673 & -2.2001 & -1.6536 \\
0.05 & -0.0428 & 0.0815 & 0.1856 & 0.5197 & -2.5501 & -2.3593 & -2.1967 & -1.6612 \\
\hline
\end{tabular}

Table 7: $l=4$ Eigenvalues for $N=2,3,4,10$

discrete cubic symmetry when $N \geq 3$. That the critical $N_{c}<3$ has been shown in very detailed multi-loop Feynman diagram calculations [29].

We may also use (3.21) and also (3.26) with (3.28) to determine $\eta^{(N)}$ when $d=3$ with the results

$$
\begin{aligned}
\eta^{(1)} & =0.0413, \quad \eta^{(2)}=0.0414, \quad \eta^{(3)}=0.0390, \quad \eta^{(4)}=0.0357 \\
\eta^{(10)} & =0.0200, \quad \eta^{(20)}=0.0125
\end{aligned}
$$

falling off as expected with increasing $N$.

In order to verify consistency with large $N$ results we also calculated eigenvalues for $N=20$ and $\eta=0$, when $k^{(20)}=3.8727448$, which are given in table 8. For $l, m$ small these are closed to the asymptotic values.

An interesting special case is $N=-2$, which was considered in [16]. For this $N$ from (3.13) we get $v^{\prime}(0)\left(v^{\prime}(0)-1+\frac{1}{2} \eta\right)=0$. When $v^{\prime}(0)=1-\frac{1}{2} \eta$ we have the high temperature 


\begin{tabular}{|c|c|r|c|c|c|c|}
\hline$m=$ & 0 & 1 & 2 & 3 & 4 & 5 \\
\hline$\lambda_{0, m}^{(20)}$ & 3.000 & 1.041 & -0.937 & -2.938 & -4.966 & -7.025 \\
$\lambda_{1, m}^{(20)}$ & 2.500 & 0.500 & -1.521 & -3.566 & -5.640 & -7.744 \\
$\lambda_{2, m}^{(20)}$ & 1.963 & -0.077 & -2.139 & -4.227 & & \\
$\lambda_{3, m}^{(20)}$ & 1.392 & -0.687 & -2.789 & -4.917 & & \\
$\lambda_{4, m}^{(20)}$ & 0.788 & -1.328 & -3.468 & -5.634 & & \\
\hline
\end{tabular}

Table 8: Eigenvalues for $N=20$

fixed point solution $v(\rho)=\left(1-\frac{1}{2} \eta\right)\left(\rho+\frac{2}{d}\right)$. The relevant non trivial fixed point arises for $v(0)=v^{\prime}(0)=0$. Consistency with our equations requires $\eta^{(-2)}=0$. To show this we may note in (3.18) that $f_{1, \phi}(\rho) \rightarrow \frac{1}{2}(2-\eta)$ as $\rho \rightarrow 0$ so that, with the definition of the scalar product in (3.20), the integral $\left\langle f_{1, \phi}, 1\right\rangle_{1}$ diverges due to the singular behaviour as $\rho \rightarrow 0$. On the other hand $\left\langle f_{1, \phi}, v^{\prime 2}\right\rangle_{1}$ and also $\left\langle f_{1, \phi}, y\right\rangle_{1}$, given by (3.28), are finite. Hence (3.26) is only consistent when $\eta=0$. For the corresponding eigenfunctions it is necessary to consider boundary conditions carefully. In (3.16) then as $\rho \rightarrow 0$ the differential equation requires solutions for $f_{l}(\rho)$ which are $\mathrm{O}(1)$ and $\mathrm{O}\left(\rho^{a}\right)$ where $a=1-\frac{1}{2}(N+2 l)-2 N k / d, k=v(0)$. Generally we impose the requirement that the second solution is absent. For $N=-2, l=0$, when also $k=0$, there are solutions with $f_{0}(\rho)=\mathrm{O}\left(\rho^{2}\right)$ for which the norm given by $(3.20)$ is finite. However to obtain eigenvalues which are related to those for $N \geq 0$ it appears necessary to consider as before solutions with $f_{0}(0)=1$, and we may impose $f_{0}{ }^{\prime \prime}(0)=0$. In this case we find numerically $\lambda_{0,1}=2$.

\section{$4 \varepsilon$ Expansion}

A further consistency check, following [21], is to consider the $\varepsilon$-expansion where

$$
d=\frac{2 n}{n-1}-\varepsilon, \quad \frac{d}{d-2}=n+\frac{1}{2}(n-1)^{2} \varepsilon+\mathrm{O}\left(\varepsilon^{2}\right), \quad n=2,3, \ldots .
$$

In this case we define

$$
x=\frac{1}{2}(d-2) \rho,
$$

and, with $v(\rho)=\hat{v}(x), \eta=0,(3.13)$ becomes

$$
x \hat{v}^{\prime \prime}(x)+(\alpha+1-x) \hat{v}^{\prime}(x)+\frac{d}{d-2} \hat{v}(x)=x \hat{v}^{\prime}(x)^{2}, \quad \alpha=\frac{1}{2} N-1 .
$$

Using (4.1) it is easy to see that as $\varepsilon \rightarrow 0$ there is a solution of the form

$$
\hat{v}(x)=k_{n} \varepsilon L_{n}^{\alpha}(x)+\mathrm{O}\left(\varepsilon^{2}\right),
$$

for $L_{n}^{\alpha}$ a Laguerre polynomial, satisfying $x L_{n}^{\alpha \prime \prime}(x)+(\alpha+1-x) L_{n}^{\alpha \prime}(x)+n L_{n}^{\alpha}(x)=0$ with the orthogonality condition

$$
\int_{0}^{\infty} \mathrm{d} x e^{-x} x^{\alpha} L_{r}^{\alpha}(x) L_{s}^{\alpha}(x)=\frac{1}{r !} \Gamma(\alpha+1+r) \delta_{r s} .
$$


To determine $k_{n}$ we consider the scalar product of both sides of (4.3) with $L_{n}^{\alpha}$ to $\mathrm{O}\left(\varepsilon^{2}\right)$ giving

$$
\frac{1}{2}(n-1)^{2} k_{n} \int_{0}^{\infty} \mathrm{d} x e^{-x} x^{\alpha} L_{n}^{\alpha}(x)^{2}=k_{n}^{2} \int_{0}^{\infty} \mathrm{d} x e^{-x} x^{\alpha+1} L_{n}^{\alpha}(x) L_{n}^{\alpha \prime}(x)^{2} .
$$

This requires

$$
k_{n}=(-1)^{n} \frac{(n-1)^{2}}{n n !} \frac{(\alpha+1)_{n}}{G_{n n n}^{(\alpha, 0)}},
$$

where we define

$$
G_{r s t}^{(\alpha, l)}=\frac{(-1)^{r+s+t}}{\Gamma(\alpha+l+1)} \int_{0}^{\infty} \mathrm{d} x e^{-x} x^{\alpha+l} L_{r}^{\alpha+l}(x) L_{s}^{\alpha+l}(x) L_{t}^{\alpha}(x) .
$$

It is straightforward to set up a perturbation expansion in $\varepsilon$ for the higher order terms in the solution (4.4) as a series summing over Laguerre polynomials $L_{r}^{\alpha}$.

For the associated eigenfunctions and eigenvalues then letting

$$
\lambda_{l, m}=d-(d-2)\left(m+\frac{1}{2} l+\hat{\lambda}_{l, m}\right),
$$

from (3.16) and (3.17) we require that $\hat{\lambda}_{l, m}$ is determined by

$$
\hat{\mathcal{D}}_{l} \hat{f}_{l, m}(x)=-\left(\hat{\lambda}_{l, m}+m\right) \hat{f}_{l, m}(x),
$$

where, with $\eta=0$ and $\alpha$ as in $(4.3), \mathcal{D}_{l}=(d-2) \hat{\mathcal{D}}_{l}$,

$$
\hat{\mathcal{D}}_{l}=x \frac{\mathrm{d}^{2}}{\mathrm{~d} x^{2}}+(\alpha+l+1-x) \frac{\mathrm{d}}{\mathrm{d} x}-2 x \hat{v}^{\prime}(x) \frac{\mathrm{d}}{\mathrm{d} x}-l \hat{v}^{\prime}(x) .
$$

Note that we must have $\hat{\lambda}_{1,0}=0, \hat{\lambda}_{1, n-1}=\varepsilon(n-1) /(d-2)$ to ensure the exact results $\lambda_{1,0}=\frac{1}{2}(d+2), \lambda_{1, n-1}=\frac{1}{2}(d-2)$. As $\varepsilon \rightarrow 0$ it is easy to see that

$$
\hat{f}_{l, m}(x) \rightarrow L_{m}^{\alpha+l}(x), \quad \hat{\lambda}_{l, m}=\mathrm{O}(\varepsilon) .
$$

To first order, where we may use (4.4) for $\hat{v}$ in (4.11), perturbation theory gives

$$
\begin{aligned}
\hat{\lambda}_{l, m} & =k_{n} \varepsilon \frac{m !}{\Gamma(\alpha+l+1+m)} \int_{0}^{\infty} \mathrm{d} x e^{-x} x^{\alpha+l} L_{m}^{\alpha+l}(x)\left(2 x L_{n}^{\alpha \prime}(x) \frac{\mathrm{d}}{\mathrm{d} x}+l L_{n}^{\alpha \prime}(x)\right) L_{m}^{\alpha+l}(x) \\
& =(-1)^{n} n k_{n} \frac{m ! G_{m m n}^{(\alpha, l)}}{(\alpha+l+1)_{m}} \varepsilon=(n-1)^{2} \frac{(\alpha+1)_{n}}{n ! G_{n n n}^{(\alpha, 0)}} \frac{m ! G_{m m n}^{(\alpha, l)}}{(\alpha+l+1)_{m}} \varepsilon .
\end{aligned}
$$

To lowest order $\eta=\mathrm{O}\left(\varepsilon^{2}\right)$. Using (3.26) and (3.28) with (4.1) and (4.4) gives

$\eta \int_{0}^{\infty} \mathrm{d} x e^{-x} x^{\alpha+1}=\frac{2 n}{2 n-1} \frac{k_{n}{ }^{2} \varepsilon^{2}}{(n-1)^{2}}\left(\int_{0}^{\infty} \mathrm{d} x e^{-x} x^{\alpha+1} L_{n}^{\alpha \prime}(x)^{2}+2 \int_{0}^{\infty} \mathrm{d} x e^{-x} x^{\alpha+2} L_{n}^{\alpha \prime \prime}(x)^{2}\right)$.

Noting that $L_{n}^{\alpha \prime}(x)=-L_{n-1}^{\alpha+1}(x)$ we then find from (4.5)

$$
\eta=\frac{2}{n !}\left(\frac{n k_{n}}{n-1}\right)^{2}(\alpha+2)_{n-1} \varepsilon^{2}=2(n-1)^{2} n !\left(\frac{(\alpha+1)_{n}}{n !^{2} G_{n n n}^{(\alpha, 0)}}\right)^{2} \varepsilon^{2} .
$$


Explicit results in (4.13) and (4.15) can be obtained using

$$
\begin{aligned}
G_{r s t}^{(\alpha, l)}= & \frac{(\alpha+l+1)_{r}(\alpha+l+1)_{s}}{(l+r+s-t) !} \\
& \times \sum_{n} \frac{1}{n !(r-n) !(s-n) !} \frac{(l+2 n) !}{(t-r-s+2 n) !} \frac{1}{(\alpha+l+1)_{n}} .
\end{aligned}
$$

From (4.16) we have $G_{00 t}^{(\alpha, l)}=\left(\begin{array}{l}l \\ t\end{array}\right)$ so that $G_{00 t}^{(\alpha, 1)}=0, t \geq 2$, and $(\alpha+1) G_{n-1 n-1 n}^{(\alpha, 1)}=\frac{1}{2} n G_{n n n}^{(\alpha, 0)}$. These ensure that $\hat{\lambda}_{1,0}=0, \hat{\lambda}_{1, n-1}=\frac{1}{2}(n-1)^{2} \varepsilon$ as required. For the case of primary relevance here $n=2$ and we have

$$
\begin{aligned}
k_{2} & =\frac{1}{2(N+8)}, \quad \eta=2 k_{2}^{2}(N+2) \varepsilon^{2}, \\
\hat{\lambda}_{l, m} & =k_{2}((l+2 m)(l+2 m-1)+m(N+2 l+2 m-2)) \varepsilon .
\end{aligned}
$$

These results are in accord with those obtained [30,31] in early calculations involving the $\varepsilon$-expansion. For the potentially marginal operators $\lambda_{4,0}=2(N-4) k_{2} \varepsilon, \lambda_{2,1}=-14 k_{2} \varepsilon$ and $\lambda_{0,2}=-\varepsilon$, so that the critical $N_{c}=4$ to leading order.

We may also extend the $O(\varepsilon)$ calculations to (3.30) for the non redundant derivative operators. With $\eta=0$ and the same change of variables as in (4.2) this becomes

$$
\begin{gathered}
\left(\hat{\mathcal{D}}_{1}-2 x \hat{v}^{\prime \prime}(x)-2 \hat{v}^{\prime}(x)\right) \hat{h}_{m}(x)=-\left(m+\hat{\lambda}_{m}\right) \hat{h}_{m}(x), \\
\lambda_{m}=-(d-2)\left(m+1+\hat{\lambda}_{m}\right), \quad m=0,1, \ldots,
\end{gathered}
$$

where with (4.4) we may take $\hat{h}_{m}(x)=L_{m}^{\alpha+1}(x)+\mathrm{O}(\varepsilon)$ and $\hat{\lambda}_{m}=\mathrm{O}(\varepsilon)$. To first order perturbation theory gives

$$
\begin{aligned}
\hat{\lambda}_{m} & =\hat{\lambda}_{1, m}+2 k_{n} \varepsilon \frac{m !}{\Gamma(\alpha+2+m)} \int_{0}^{\infty} \mathrm{d} x e^{-x} x^{\alpha+1} L_{m}^{\alpha+1}(x)^{2}\left(x L_{n}^{\alpha \prime \prime}(x)+L_{n}^{\alpha \prime}(x)\right) \\
& =\hat{\lambda}_{1, m}+(-1)^{n} 2 k_{n} \varepsilon \frac{m !}{(\alpha+2)_{m}}\left((n+\alpha) G_{m m n-1}^{(\alpha, 1)}-\alpha G_{m m n-1}^{(\alpha+1,0)}\right)
\end{aligned}
$$

using the identity $x L_{n}^{\alpha \prime \prime}(x)+L_{n}^{\alpha \prime}(x)=-(n+\alpha) L_{n-1}^{\alpha}(x)+\alpha L_{n-1}^{\alpha+1}(x)$. When $n=2$ this gives

$$
\hat{\lambda}_{m}=\hat{\lambda}_{1, m}+k_{2} \varepsilon(N+8 m+2), \quad \hat{\lambda}_{1, m}=k_{2} \varepsilon m(N+6 m+2),
$$

giving

$$
\hat{\lambda}_{m}=\frac{(m+1) N+6 m^{2}+10 m+2}{2(N+8)} \varepsilon .
$$

\section{Perturbative Discussion}

The crucial significance of the $\varepsilon$-expansion is that it is possible to use standard perturbative methods in quantum field theory involving a Feynman graph expansion. To show the 
parallel with the above treatment we describe how the same results are found in terms of conventional $\beta$-functions. For a general scalar lagrangian

$$
\mathcal{L}=\frac{1}{2} \partial^{\mu} \phi \cdot \partial_{\mu} \phi+V(\phi),
$$

then we may define a generalised $\beta$-function for the potential $V$, which is a linear function of the couplings ${ }^{1}$, where

$$
B_{V}(\phi)=\frac{1}{2}(d-2) \phi \cdot \partial V(\phi)-d V(\phi)+\beta_{V}(\phi) .
$$

The perturbative RG flow equations are then

$$
\dot{V}(\phi)=-B_{V}(\phi),
$$

and the fixed points $V=V_{*}$ are hence defined by

$$
B_{V_{*}}(\phi)=0 .
$$

With $d$ as in (4.1) then for a renormalisable theory $V(\phi)$ is a polynomial of degree $2 n$. Using background field methods and minimal subtraction [32] then in a perturbative expansion calculations give $\beta_{V}$ in the form

$$
\beta_{V}(\phi)=\left(\gamma_{\phi} \phi\right) \cdot \partial V(\phi)+\tilde{\beta}_{V}(\phi),
$$

where $\gamma_{\phi, i j}$ is the anomalous dimension matrix for the field $\phi$ and $\tilde{\beta}_{V}(\phi)$ depends on $\phi$ solely in terms of scalar contractions of various products of $V_{i_{1} i_{2} \ldots i_{k}}(\phi)=\partial_{i_{1}} \ldots \partial_{i_{k}} V(\phi)$ with $k \geq 2$. For renormalisable $V, \gamma_{\phi}$ depends only on the $\phi$-independent $g_{i_{1} i_{2} \ldots i_{2 n}}=V_{i_{1} i_{2} \ldots i_{2 n}}$ and $\beta_{V}(\phi)$ is also just a polynomial of degree $2 n$. In general there are contributions to $\beta_{V}$ at $(p-1)(n-1)$ loops, $p=2,3 \ldots$, when $\tilde{\beta}_{V}=\mathrm{O}\left(\partial^{2 n(p-1)} V^{p}\right)$ and, if $p>2, \gamma_{\phi}(g)=\mathrm{O}\left(g^{p-1}\right)$.

Assuming $V_{*}(\phi)=\frac{1}{(2 n) !} g_{* i_{1} i_{2} \ldots i_{2 n}} \phi_{i_{1}} \cdots \phi_{i_{2 n}}$ then (5.4) determines, for $n=2,3, \ldots$, $g_{* i_{1} i_{2} \ldots i_{2 n}}$ perturbatively as an expansion in $\varepsilon$. At the fixed point the anomalous dimension matrix for $\phi$ also defines

$$
\eta_{i j}=\left.2 \gamma_{\phi, i j}\right|_{g=g_{*}}
$$

In the vicinity of a fixed point defining, for $F(\phi)$ an arbitrary polynomial of degree $2 n$, a linear operator $\gamma$ by

$$
\beta_{V+F}(\phi)=\beta_{V}(\phi)+\gamma F(\phi)+\mathrm{O}\left(F^{2}\right),
$$

then critical exponents are determined by the eigenvalue equation

$$
\Delta F(\phi)=\frac{1}{2}(d-2) \phi \cdot \partial F(\phi)+\gamma_{*} F(\phi)=(d-\lambda) F(\phi), \quad \gamma_{*}=\left.\gamma\right|_{V=V_{*}} .
$$

As in the discussion of the exact RG equations there are explicit eigenfunctions relating to $\phi$ itself. Corresponding to (5.5) we have

$$
\gamma=\left(\gamma_{\phi} \phi\right) \cdot \partial+\tilde{\gamma},
$$

\footnotetext{
${ }^{1}$ For $V(\phi)=\sum_{I} g^{I} \mathcal{O}_{I}$, where $\mathcal{O}^{I}$ form a basis of monomials in $\phi$, then $\beta_{V}(\phi)=\sum_{I} \beta^{I}(g) \mathcal{O}_{I}$. Subsequently $\beta_{V} \cdot \frac{\partial}{\partial V}=\sum_{I} \beta^{I}(g) \frac{\partial}{\partial g^{I}}$.
} 
where $\tilde{\gamma}$ involves at least second order $\phi$-derivatives. Hence

$$
\Delta \phi_{i}=\frac{1}{2}(d-2) \phi_{i}+\frac{1}{2} \eta_{i j} \phi_{j},
$$

and also by differentiating (5.4)

$$
\Delta V_{*, i}(\phi)=\frac{1}{2}(d+2) V_{*, i}(\phi)-\frac{1}{2} \eta_{i j} V_{*, j}(\phi) .
$$

$\mathcal{L}$ is arbitrary up to total derivatives so in this discussion mixing with operators which are just spatial derivatives is neglected. The relevant matrix is triangular so the determination of scale dimensions is not affected. Up to a derivative operator proportional to $\partial^{2} \phi_{i}, V_{*, i}(\phi) \sim$ 0 , as a consequence of the field equations so this is redundant.

At lowest order, as shown using background field techniques with $n-1$ loop Feynman diagrams in [21],

$$
\beta_{V}(\phi)^{(n-1)}=a_{n} V_{i_{1} \cdots i_{n}}(\phi) V_{i_{1} \cdots i_{n}}(\phi),
$$

where higher loops are $\mathrm{O}\left(V^{3}\right)$. Also, for $2(n-1)$ loops,

$$
\gamma_{\phi, i j}{ }^{(2 n-2)}=2 \frac{(n !)^{2}}{(2 n) !} a_{n}{ }^{2} g_{i i_{1} i_{2} \ldots i_{2 n-1}} g_{j i_{1} i_{2} \ldots i_{2 n-1}},
$$

where

$$
a_{n}=\frac{1}{(4 \pi)^{n}} \frac{n-1}{n !} \Gamma\left(\frac{1}{n-1}\right)^{n-1} .
$$

The definition (5.7) and (5.12), then determines $\mathcal{D}_{V}$ to lowest order

$$
\tilde{\gamma}^{(n-1)}=2 a_{n} V_{i_{1} \cdots i_{n}}(\phi) F_{i_{1} \cdots i_{n}}(\phi) .
$$

As before we impose $O(N)$ symmetry so that

$$
V(\phi)=g \frac{1}{n !}\left(\frac{1}{2} \phi^{2}\right)^{n}, \quad \beta_{V}(\phi)=\beta_{g}(g) \frac{1}{n !}\left(\frac{1}{2} \phi^{2}\right)^{n}, \quad \gamma_{\phi, i j}=\gamma_{\phi}(g) \delta_{i j} .
$$

At the fixed point (5.4)

$$
\beta_{g}\left(g_{*}\right)=(n-1) g_{*} \varepsilon, \quad \eta=2 \gamma_{\phi}\left(g_{*}\right) .
$$

With $O(N)$ symmetry the eigenfunctions in (5.8) have the form

$$
F_{l, m}(\phi)=(t \cdot \phi)^{l}\left(\frac{1}{2} \phi^{2}\right)^{m}, \quad t^{2}=0 .
$$

The corresponding eigenvalues are then

$$
d-\lambda_{l, m}=\frac{1}{2}(d-2+\eta)(l+2 m)+\gamma_{l, m},
$$

with $\gamma_{l, m}$ the anomalous dimension determined by $\tilde{\gamma}_{*} F_{l, m}(\phi)=\gamma_{l, m} F_{l, m}(\phi)$.

To handle the combinatorics involved in evaluating (5.15) with $F$ as in (5.18) we reexpress this using

$$
V_{i_{1} \cdots i_{n}}(\phi) F_{i_{1} \cdots i_{n}}(\phi)=\left.\left(\frac{\partial}{\partial \phi} \cdot \frac{\partial}{\partial \phi^{\prime}}\right)^{n}\left(V(\phi) F\left(\phi^{\prime}\right)\right)\right|_{\phi^{\prime}=\phi},
$$


and then follow the method described in [31]. First we note

$$
(a \cdot \partial)^{k}\left(\frac{1}{2} \phi^{2}\right)^{n}=\sum_{r} \frac{k ! n !}{r !(k-2 r) !(n-k+r) !}\left(\frac{1}{2} \phi^{2}\right)^{n-k+r}\left(\frac{1}{2} a^{2}\right)^{r}(a \cdot \phi)^{k-2 r},
$$

and then

$$
\begin{aligned}
& \left(\frac{\partial}{\partial \phi} \cdot \frac{\partial}{\partial \phi^{\prime}}\right)^{k}\left(\left(\frac{1}{2} \phi^{2}\right)^{n}\left(t \cdot \phi^{\prime}\right)^{l}\left(\frac{1}{2} \phi^{\prime 2}\right)^{m}\right) \\
& \quad=\sum_{r} \frac{k ! n !}{r !(k-2 r) !(n-k+r) !}\left(\frac{1}{2} \phi^{2}\right)^{n-k+r}\left(\phi \cdot \partial^{\prime}\right)^{k-2 r}\left(\frac{1}{2} \partial^{\prime 2}\right)^{r}\left(\left(t \cdot \phi^{\prime}\right)^{l}\left(\frac{1}{2} \phi^{\prime 2}\right)^{m}\right) .
\end{aligned}
$$

Using

$$
\left(\frac{1}{2} \partial^{\prime 2}\right)^{r}\left(\left(t \cdot \phi^{\prime}\right)^{l}\left(\frac{1}{2} \phi^{\prime 2}\right)^{m}\right)=\frac{m !}{(m-r) !}(\alpha+l+1+m-r)_{r}\left(t \cdot \phi^{\prime}\right)^{l}\left(\frac{1}{2} \phi^{\prime 2}\right)^{m-r},
$$

with $\alpha$ as in (4.3), and

$$
\left.\left(\phi \cdot \partial^{\prime}\right)^{p}\left(\left(t \cdot \phi^{\prime}\right)^{l}\left(\frac{1}{2} \phi^{\prime 2}\right)^{m}\right)\right|_{\phi^{\prime}=\phi}=\frac{(2 m+l) !}{(2 m+l-p) !}(t \cdot \phi)^{l}\left(\frac{1}{2} \phi^{2}\right)^{m},
$$

we then obtain ${ }^{2}$

$$
\begin{aligned}
& \left.\frac{1}{n !}\left(\frac{\partial}{\partial \phi} \cdot \frac{\partial}{\partial \phi^{\prime}}\right)^{k}\left(\left(\frac{1}{2} \phi^{2}\right)^{n}\left(t \cdot \phi^{\prime}\right)^{l}\left(\frac{1}{2} \phi^{2}\right)^{m}\right)\right|_{\phi^{\prime}=\phi}=A_{k n, m}^{(\alpha, l)}(t \cdot \phi)^{l}\left(\frac{1}{2} \phi^{2}\right)^{m+n-k} \\
& A_{k n, m}^{(\alpha, l)}=\sum_{r} \frac{k !}{r !(k-2 r) !(n-k+r) !} \frac{m !}{(m-r) !}(\alpha+l+1+m-r)_{r} \frac{(2 m+l-2 r) !}{(2 m+l-k) !}
\end{aligned}
$$

Hence from (5.12) and (5.15) with (5.16) and (5.18) we may obtain to lowest order

$$
\beta_{g}(g)^{(n-1)}=A_{n n, n}^{(\alpha, 0)} a_{n} g^{2},
$$

From the fixed point equation (5.17), with $\eta=0$ and (5.26), we get

$$
A_{n n, n}^{(\alpha, 0)} a_{n} g_{*}=(n-1) \varepsilon .
$$

In consequence

$$
\tilde{\gamma}_{*}^{(n-1)} F_{l, m}(\phi)=2 a_{n} g_{*} A_{n, m}^{(\alpha, l)} F_{l, m}(\phi) .
$$

and, in (5.8) and (5.19), to lowest order

$$
\gamma_{l, m}=2 a_{n} g_{*} A_{n n, m}^{(\alpha, l)}=2(n-1) \frac{A_{n n, m}^{(\alpha, l)}}{A_{n n, n}^{(\alpha, 0)}} \varepsilon .
$$

${ }^{2}$ The coefficients $A_{k n, m}^{(\alpha, l)}$ satisfy various identities, in particular

$$
\begin{aligned}
& (\alpha+l)\left(A_{k+1 n, m}^{(\alpha, l)}+2 m(\alpha+l+m) A_{k n, m-1}^{(\alpha, l)}\right) \\
& \quad=m(2 \alpha+l)(\alpha+l+m+n-k) A_{k n, m-1}^{(\alpha, l+1)}+l(\alpha+l+m)(m+n-k) A_{k n, m}^{(\alpha, l-1)} .
\end{aligned}
$$

When $N=1, A_{k n, m}^{\left(-\frac{1}{2}, l\right)}=\frac{(2 n) !(2 m+l) !}{2^{k} n !(2 n-k) !(2 m+l-k) !}$ for $l=0,1$. 
Note that $\gamma_{0, n}=\beta_{g}^{\prime}\left(g_{*}\right)$.

As a special case of (5.25) we also have

$$
\left.\frac{\partial}{\partial \phi_{i}} \frac{\partial}{\partial \phi_{j}^{\prime}}\left(\frac{\partial}{\partial \phi} \cdot \frac{\partial}{\partial \phi^{\prime}}\right)^{2 n-1}\left(\left(\frac{1}{2} \phi^{2}\right)^{n}\left(\frac{1}{2} \phi^{\prime 2}\right)^{n}\right)\right|_{\phi^{\prime}=\phi}=\frac{1}{2}(2 n) ! n !(\alpha+2)_{n-1} \delta_{i j}
$$

so that (5.6) and (5.13) give

$$
\eta=2 n !(\alpha+2)_{n-1}\left(a_{n} g_{*}\right)^{2}=2(n-1)^{2} n ! \frac{1}{\left(A_{n n, n}^{(\alpha, 0)}\right)^{2}} \varepsilon^{2} .
$$

The precise identity of the results (5.29) and (5.31) with those obtained from the RG equation (4.13) and (4.15), where $\hat{\lambda}_{l, m}=\frac{1}{2}(n-1) \gamma_{l, m}$, follows from

$$
A_{k n, m}^{(\alpha, l)}=k ! m ! \frac{G_{m+n-k m n}^{(\alpha, l)}}{(\alpha+l+1)_{m+n-k}} .
$$

When $n=2$ the $\mathrm{O}(\varepsilon)$ results may be read off from

$$
2 a_{2} g_{*}=\frac{\varepsilon}{N+8}, \quad A_{22, m}^{(\alpha, l)}=(2 m+l)(2 m+l-1)+m(N+2 l+2 m-2) .
$$

\section{$6 \quad$ Mixing Effects}

For operators which are monomials $\phi^{m}$ with $m \geq 2 n$ then perturbatively it is necessary to include mixing effects with operators $(\partial \phi)^{2} \phi^{m-2 n}$. For $m \geq 4 n-2$ there is additional mixing with operators involving four derivatives, such as $\left(\partial^{2} \phi\right)^{2}$, but this is neglected here. We here discuss how the treatment of the previous section may be extended and show how reparameterisation invariance is manifest in a perturbative approach.

The initial renormalisable lagrangian $\mathcal{L}$ in $(5.1)$ is extended to

$$
\mathcal{L}=\mathcal{L}_{V}+\mathcal{L}_{F, G}, \quad \mathcal{L}_{V}=\frac{1}{2} \partial^{\mu} \phi \cdot \partial_{\mu} \phi+V(\phi), \quad \mathcal{L}_{F, G}=F(\phi)+\frac{1}{2} G_{i j}(\phi) \partial^{\mu} \phi_{i} \partial_{\mu} \phi_{j} .
$$

Although for general $F, G_{i j}$ the resulting $\mathcal{L}$ is non renormalisable, keeping only counterterms which are linear in $F, G_{i j}$, we may consistently define a bare lagrangian $\mathcal{L}_{0}$ which extends the renormalisable theory defined by $\mathcal{L}_{V}$ to include first order perturbations by finite two derivative operators, so long as $F(\phi), G_{i j}(\phi)$ are constrained to avoid the necessity of four derivative counterterms. As usual there are corresponding $\beta$-functions

$$
\begin{aligned}
B_{F}(\phi) & =\frac{1}{2}(d-2) \phi \cdot \partial F(\phi)-d F(\phi)+\beta_{F}(\phi), \\
B_{G, i j}(\phi) & =\frac{1}{2}(d-2) \phi \cdot \partial G_{i j}(\phi)+\gamma_{\phi, i k} G_{k j}(\phi)+\gamma_{\phi, j k} G_{i k}(\phi)+\beta_{G, i j}(\phi),
\end{aligned}
$$

which are linear in $F, G_{i j}$ so that

$$
\beta_{F}=\gamma_{F F} F+\gamma_{F G, i j} G_{i j}, \quad \beta_{G, i j}=\gamma_{G F, i j} F+\gamma_{G G, i j k l} G_{k l} .
$$


Here $\gamma_{F F}, \gamma_{F G, i j}, \gamma_{G F, i j}, \gamma_{F G, i j k l}$ are differential operators depending on the renormalisable couplings or $V$, clearly we have, restricted to $F(\phi)$ of degree $2 n, \gamma_{F F}=\gamma$ as defined in (5.7). At a fixed point the exponents are defined by the coupled equations

$$
\left.B_{F}(\phi)\right|_{V=V_{*}}=-\lambda F(\phi),\left.\quad B_{G, i j}(\phi)\right|_{V=V_{*}}=-\lambda G_{i j}(\phi) .
$$

For the lagrangians in (6.1) we have

$$
\delta \mathcal{L}_{V}=\delta_{F, G} \mathcal{L}_{F, G} \quad \text { for } \quad \delta \phi_{i}=v_{i}(\phi), \quad \delta \partial_{\mu} \phi_{i}=v_{i, j}(\phi) \partial_{\mu} \phi_{j},
$$

if $^{3}$

$$
\delta_{F, G} F(\phi)=v(\phi) \cdot \partial V(\phi), \quad \delta_{F, G} G_{i j}(\phi)=\partial_{i} v_{j}(\phi)+\partial_{j} v_{i}(\phi) .
$$

If $F(\phi), G_{i j}(\phi)$ are restricted to ensure that no mixing with four derivative operators arises then it is necessary to require $v_{i}(\phi)=\mathrm{O}\left(\phi^{2(n-1)}\right)$.

As was apparent in the discussion of renormalisation for general two dimensional $\sigma$ models $[33,34]$ invariance under reparameterisations $\delta \phi_{i}=v_{i}(\phi)$ leads to a corresponding freedom in the definition of the $\beta$-functions. Here we show how this leads to relations for the exponents defined by (6.4). Assuming first

$$
\begin{aligned}
\left.\beta_{F}(\phi)\right|_{F=v \cdot \partial V, G_{i j}=\partial_{i} v_{j}+\partial_{j} v_{i}} & =(\gamma v(\phi)) \cdot \partial V(\phi)+v(\phi) \cdot \partial \beta_{V}(\phi), \\
\left.\beta_{G, i j}(\phi)\right|_{F=v \cdot \partial V, G_{k l}=\partial_{k} v_{l}+\partial_{l} v_{k}} & =\partial_{i}\left(\gamma v_{j}(\phi)-2 \gamma_{\phi, j k} v_{k}(\phi)\right)+\partial_{j}\left(\gamma v_{i}(\phi)-2 \gamma_{\phi, i k} v_{k}(\phi)\right),
\end{aligned}
$$

with $\gamma$ defined in (5.8), then (6.2) gives

$$
\begin{aligned}
\left.B_{F}(\phi)\right|_{F=v \cdot \partial V, G_{i j}=\partial_{i} v_{j}+\partial_{j} v_{i}} & =U(\phi) \cdot \partial V(\phi)+v(\phi) \cdot \partial B_{V}(\phi), \\
\left.B_{G, i j}(\phi)\right|_{F=v \cdot \partial V, G_{k l}=\partial_{k} v_{l}+\partial_{l} v_{k}} & =\partial_{i} U_{j}(\phi)+\partial_{j} U_{i}(\phi),
\end{aligned}
$$

for

$$
U_{i}(\phi)=\frac{1}{2}(d-2) \phi \cdot \partial v_{i}(\phi)+\gamma v_{i}(\phi)-\frac{1}{2}(d-2) v_{i}(\phi)-\gamma_{\phi, i j} v_{j}(\phi) .
$$

A general justification of (6.8a),(6.8b) with (6.9) is described in appendix C.

At a critical point, where (5.4) holds, then for vector solutions of (5.8), $\Delta v_{i}=\left(d-\lambda_{v}\right) v_{i}$, there are, as a consequence of $(6.8 \mathrm{a}),(6.8 \mathrm{~b})$ corresponding solutions of (6.4) such that

$$
\lambda=\lambda_{v}-\frac{1}{2}(d+2-\eta),
$$

assuming a diagonal form for $\eta_{i j}$. Thus in this perturbative context we reproduce (1.1). In particular as a consequence of (5.10) we have the exact zero modes

$$
F_{0}(\phi)=\phi \cdot \partial V(\phi), \quad G_{0, i j}=2 \delta_{i j} .
$$

\footnotetext{
${ }^{3}$ The full Lagrangian in (6.1) is invariant if (6.6) is extended to $\delta F=v \cdot \partial(V+F), \delta G_{i j}=\partial_{i} v_{j}+\partial_{j} v_{i}+$ $v \cdot \partial G_{i j}+\partial_{i} v_{k} G_{k j}+\partial_{j} v_{k} G_{i k}$.
} 
To verify these results we consider the lowest order perturbative results at $n-1$ loops

$$
\begin{aligned}
\beta_{F}(\phi)^{(n-1)}= & 2 a_{n} V_{i_{1} \ldots i_{n}}(\phi) F_{i_{1} \ldots i_{n}}(\phi) \\
& -a_{n} \sum_{\substack{r, s, t \geq 1 \\
r+s+t=n+1}} \frac{n !}{r ! s ! t !} \hat{K}_{r s t} V_{i_{1} \ldots i_{r} k_{1} \ldots k_{t}}(\phi) V_{j_{1} \ldots j_{s} k_{1} \ldots k_{t}}(\phi) G_{i_{1} j_{1}, i_{2} \ldots i_{r} j_{2} \ldots j_{s}}(\phi) \\
& +a_{n} \sum_{\substack{r \geq 2, s, t \geq 1 \\
r+s+t=n+1}} \frac{n !}{r ! t} \hat{K}_{r s t} V_{i_{1} \ldots i_{r} k_{1} \ldots k_{t}}(\phi) V_{j_{1} \ldots j_{s} k_{1} \ldots k_{t}}(\phi) G_{i_{1} i_{2}, i_{3} \ldots i_{r} j_{1} \ldots j_{s}}(\phi),
\end{aligned}
$$

where $a_{n}$ is as in (5.14) and

$$
\hat{K}_{r s t}=\Gamma\left(\frac{1}{n-1}\right) \frac{\Gamma\left(\frac{n-r}{n-1}\right) \Gamma\left(\frac{n-s}{n-1}\right) \Gamma\left(\frac{n-t}{n-1}\right)}{\Gamma\left(\frac{r}{n-1}\right) \Gamma\left(\frac{s}{n-1}\right) \Gamma\left(\frac{t}{n-1}\right)} .
$$

When $r=1, s+t=n, \hat{K}_{r s t}=1$. Also

$$
\begin{gathered}
\beta_{G, i j}(\phi)^{(n-1)}=2 a_{n}\left(V_{i_{1} \ldots i_{n}}(\phi) G_{i j, i_{1} \ldots i_{n}}(\phi)+2 V_{i_{1} \ldots i_{n}(i}(\phi) G_{j) i_{1}, i_{2} \ldots i_{n}}(\phi)\right. \\
\left.-V_{i_{1} \ldots i_{n}(i}(\phi) G_{\left.i_{1} i_{2}, j\right) i_{3} \ldots i_{n}}(\phi)\right) .
\end{gathered}
$$

We may directly verify that (6.12) and (6.14) satisfy (6.7a),(6.7b) with $\tilde{\gamma}^{(n-1)}$ given by (5.15) and also $\gamma_{\phi}=0$. At the order given in (6.14) there are no contributions involving $F$. At the next non zero order

$$
\begin{aligned}
\beta_{G, i j}(\phi)^{(2 n-2)}= & -8 a_{n}{ }^{2} \frac{(n !)^{2}}{(2 n) !} g_{i_{1} \ldots i_{2 n-1}(i} F_{j) i_{1} \ldots i_{2 n-1}}(\phi) \\
& +(2 n-1) 4 a_{n}{ }^{2} \frac{(n !)^{2}}{(2 n) !} g_{i_{1} \ldots i_{2 n-2} k(i} g_{\left.i_{1} \ldots i_{2 n-2} l j\right)} G_{k l}(\phi)+\mathrm{O}(\partial G),
\end{aligned}
$$

which is also compatible with (6.7a),(6.7b) using (5.13) for $\gamma_{\phi}$.

For definite results we assume $O(N)$ symmetry as in (5.16). To first order in $\varepsilon$ the equations (6.4) decouple and we may write the eigenvalue equation

$$
\frac{1}{2}(d-2) \phi \cdot \partial G_{i j}(\phi)+2 a_{n} g_{*} \mathcal{D} G_{i j}(\phi)=-\lambda G_{i j}(\phi),
$$

where from (6.14) $\mathcal{D} G_{i j}$ is given by

$$
\begin{aligned}
\mathcal{D} G_{i j}(\phi)= & \left.\frac{1}{n !}\left(\frac{\partial}{\partial \phi} \cdot \frac{\partial}{\partial \phi^{\prime}}\right)^{n}\left(\left(\frac{1}{2} \phi^{2}\right)^{n} G_{i j}\left(\phi^{\prime}\right)\right)\right|_{\phi^{\prime}=\phi}+\left.\frac{2}{n !}\left(\frac{\partial}{\partial \phi} \cdot \frac{\partial}{\partial \phi^{\prime}}\right)^{n-1}\left(\partial_{k} \partial_{(i}\left(\frac{1}{2} \phi^{2}\right)^{n} G_{j) k}\left(\phi^{\prime}\right)\right)\right|_{\phi^{\prime}=\phi} \\
& -\left.\frac{1}{n !}\left(\frac{\partial}{\partial \phi} \cdot \frac{\partial}{\partial \phi^{\prime}}\right)^{n-2}\left(\partial_{k} \partial_{l} \partial_{(i}\left(\frac{1}{2} \phi^{2}\right)^{n} \partial_{j)}^{\prime} G_{k l}\left(\phi^{\prime}\right)\right)\right|_{\phi^{\prime}=\phi} .
\end{aligned}
$$

Clearly for $G_{i j}(\phi)=\delta_{i j}$ then $\lambda=0$. In general we have

$$
\begin{aligned}
\mathcal{D}\left(\phi_{i} \phi_{j}\left(\frac{1}{2} \phi^{2}\right)^{m}\right)= & \left.\frac{1}{m+1} \partial_{(i} \frac{1}{n !}\left(\frac{\partial}{\partial \phi} \cdot \frac{\partial}{\partial \phi^{\prime}}\right)^{n}\left(\left(\frac{1}{2} \phi^{2}\right)^{n} \phi_{j)}^{\prime}\left(\frac{1}{2} \phi^{2}\right)^{m+1}\right)\right|_{\phi^{\prime}=\phi} \\
& -\frac{1}{m+1} \mathcal{D}\left(\delta_{i j}\left(\frac{1}{2} \phi^{2}\right)^{m+1}\right),
\end{aligned}
$$


so that from $(5.25)$

$$
\begin{aligned}
G_{i j}(\phi) & =\delta_{i j}\left(\frac{1}{2} \phi^{2}\right)^{m+1}+(m+1) \phi_{i} \phi_{j}\left(\frac{1}{2} \phi^{2}\right)^{m}, \quad m=0,1, \ldots, \\
\Rightarrow \quad \lambda_{m} & =-(d-2)(m+1)-2 a_{n} g_{*} A_{n n, m+1}^{(\alpha, 1)}=-(d-2)(m+1)-\gamma_{1, m+1},
\end{aligned}
$$

as expected from the general relation (6.10) with (5.19) and $\eta=0$. In general

$$
\frac{1}{m+1} \mathcal{D}\left(\delta_{i j}\left(\frac{1}{2} \phi^{2}\right)^{m+1}\right)=\alpha_{m} \delta_{i j}\left(\frac{1}{2} \phi^{2}\right)^{m+1}+\beta_{m} \phi_{i} \phi_{j}\left(\frac{1}{2} \phi^{2}\right)^{m}
$$

and we then find another eigenvalue, in addition to (6.19), which can ne expressed in the form

$$
\lambda_{m}=-(d-2)(m+1)-\gamma_{m}, \quad \gamma_{m}=2 a_{n} g_{*}\left((m+1) \alpha_{m}-\beta_{m}\right) .
$$

To obtain more explicit results for this we apply (6.17) to obtain

$$
\begin{aligned}
\mathcal{D}\left(\delta_{i j}\left(\frac{1}{2} \phi^{2}\right)^{m}\right)= & A_{n, m}^{(\alpha, 0)} \delta_{i j}\left(\frac{1}{2} \phi^{2}\right)^{m} \\
& +2\left((m+1) A_{n-1 n, m}^{(\alpha, 0)}-2 m A_{n-1 n, m-1}^{(\alpha, 1)}-(\alpha+n) m A_{n-2 n-1, m-1}^{(\alpha, 1)}\right) \\
& \quad \times\left(\delta_{i j}\left(\frac{1}{2} \phi^{2}\right)^{m}+m \phi_{i} \phi_{j}\left(\frac{1}{2} \phi^{2}\right)^{m-1}\right) \\
& +\left.\frac{2}{n !}\left(\frac{\partial}{\partial \phi} \cdot \frac{\partial}{\partial \phi^{\prime}}\right)^{n-1}\left(\left(\frac{1}{2} \phi^{2}\right)^{n} \partial_{i}^{\prime} \partial_{j}^{\prime}\left(\frac{1}{2} \phi^{\prime 2}\right)^{m}\right)\right|_{\phi^{\prime}=\phi} \\
& +\left.(\alpha+n) \frac{2}{(n-1) !}\left(\frac{\partial}{\partial \phi} \cdot \frac{\partial}{\partial \phi^{\prime}}\right)^{n-2}\left(\left(\frac{1}{2} \phi^{2}\right)^{n-1} \partial_{i}^{\prime} \partial_{j}^{\prime}\left(\frac{1}{2} \phi^{\prime 2}\right)^{m}\right)\right|_{\phi^{\prime}=\phi} .
\end{aligned}
$$

From this we may determine

$$
\begin{aligned}
(m+1) \alpha_{m}-\beta_{m}= & A_{n n, m+1}^{(\alpha, 0)} \\
& +\frac{4}{N}(m+1)(\alpha+m+1)\left(A_{n-1 n, m}^{(\alpha, 0)}+(\alpha+n) A_{n-2 n-1, m}^{(\alpha, 0)}\right) \\
& -\frac{4}{N} m(\alpha+m+2)\left(A_{n-1 n, m-1}^{(\alpha, 2)}+(\alpha+n) A_{n-2 n-1, m-1}^{(\alpha, 2)}\right) .
\end{aligned}
$$

Combining (6.23) with (6.21) gives the exponents for new non redundant operators so long as $N \geq 2$, for $N=1$ the corresponding eigenfunction vanishes. When $n=2$ we have

$$
(m+1) \alpha_{m}-\beta_{m}=(m+2) N+6 m^{2}+12 m+4 .
$$

Hence combining this with (5.33) the anomalous dimensions of these derivative operators at the fixed point, to first order in $\varepsilon$, are given by

$$
\gamma_{m}=\frac{(m+2) N+6 m^{2}+12 m+4}{N+8} \varepsilon .
$$

This may be compared with $2 \hat{\lambda}_{m}$ in (4.21) which was obtained from the approximate derivative expansion for the Polchinski RG equations. Although similar they are not identical. The perturbative results in (6.25) are of course the first terms in a well defined expansion to any order in powers of $\varepsilon$. 


\section{Conclusion}

The status of the equations presented in this paper for extending the local potential approximation to the Polchinski exact RG equation is unclear, in that there is no consistent derivation and the resulting equations for $V, Z$ are partially decoupled in that the $V$-equation lacks expected $Z$ contributions. Nevertheless the LPA equation now involves $\eta$ which, in this respect, is similar to an approximation made in a treatment of exact $R G$ equations in [35]. It remains to be seen whether the introduction of terms involving $Z$ into the Polchinski LPA equations is at all possible, while maintaining the crucial property of reparameterisation invariance, and so allowing a well defined determination of $\eta$. Some time ago Morris [16] obtained, with a particular cut off function $K\left(p^{2}\right)$ proportional to a simple power of $p^{2}$, and for the exact RG equations applicable to the one particle irreducible functional $\Gamma$, a set of equations, in a derivative expansion, which are invariant under global rescaling of the fields. To this extent reparameterisation invariance is preserved and there is consequently an exact zero mode so that $\eta$ may be determined unambiguously. However these equations are highly nonlinear and hard to analyse. The associated zero mode eigenfunction for these equations has not apparently been found explicitly in the literature. If a derivative expansion is to be consistent then results should not change dramatically when the LPA for $V$ is extended to first order in derivative operators to a pair of coupled equations for $V, Z$. However results for some eigenvalues obtained using the Morris equations differ significantly [26]. It would be very desirable to understand more analytically what features of the equations in [16] ensure reparameterisation invariance so that this crucial constraint might be imposed more generally in derivative expansion RG equations. It might also be helpful, as was the case here, to construct an appropriate scalar product for the eigenfunctions whose corresponding eigenvalues are the essential output of exact RG equations.

In general reparameterisation invariance, which is related to issues of scheme independence, is akin to a gauge invariance of the exact RG equations [36]. As is well known for gauge theories violating gauge invariance in some approximation can lead to unphysical conclusions. Emphasising the consequences of reparameterisation invariance, and the consequential presence of an exact zero mode in the RG flow equations, might also be a useful criteria for optimisation in equations where reparameterisation invariance is not automatic.

It is of course non trivial that the LPA captures the global aspects of RG flows between various possible fixed points in scalar field theories. The calculated critical exponents are also of essentially the correct magnitude since the results must agree with those from ordinary perturbation theory for non derivative operators to first order in $\varepsilon$ (strictly this appears to have been shown only when maximal $O(N)$ symmetry is required, it is presumably true for fixed points with lesser symmetry although a general argument appears to be lacking). A natural constraint for any derivative expansion is that this agreement should extend to non redundant scalar operators with two derivatives. The discrepancy between the anomalous dimensions for such operators given by (4.21) and (6.25) shows that the equations discussed here are not fully satisfactory in that respect. Perhaps more general derivative expansion equations can be obtained by using more input from perturbative results.

\section{Acknowledgements}


HO would like to thank Claude Bervillier and Oliver Rosten for valuable email correspondence. DET would like to thank Trinity College for a summer research studentship. 


\section{A Large $N$ Calculations}

Solutions of the LPA fixed point equations become more tractable in the large $N$ limit $[27,37]$. We show here how these results may be used to obtain a corresponding value for $\eta$ using (3.21) or (3.26) and (3.28). In the large $N$ limit we suppose $v, \rho=\mathrm{O}(N)$ and taking the derivative of (3.13) then gives

$$
\left((d-2+\eta) \rho-N+4 \rho v^{\prime}\right) v^{\prime \prime}=(2-\eta) v^{\prime}-2 v^{\prime 2},
$$

or

$$
2 v^{\prime}\left(s-v^{\prime}\right) \frac{\mathrm{d} \rho}{\mathrm{d} v^{\prime}}-\left(d-2 s+4 v^{\prime}\right) \rho=-N, \quad s=1-\frac{1}{2} \eta .
$$

Such first order linear equations are readily solved giving $\rho$ as a function of $v^{\prime}$,

$$
\frac{\left(s-v^{\prime}\right)^{\frac{1}{2 s} d+1}}{v^{\prime} \frac{1}{2 s} d-1} \rho=C-\frac{1}{2} N \int_{0}^{v^{\prime}} \mathrm{d} u\left(\frac{s-u}{u}\right)^{\frac{1}{2 s} d},
$$

with the integral extended by analytic continuation for $\frac{1}{2 s} d>1$. To ensure a smooth continuously differentiable solution for all $\rho>0$ we must set the constant of integration $C=0$ and then the large $N$ result can be expressed as

$$
\rho=\frac{N}{d-2 s} F\left(2,1 ; 2-\frac{1}{2 s} d ; \frac{1}{s} v^{\prime}\right)
$$

for $F$ a standard hypergeometric function.

In the large $N$ limit $\eta \rightarrow 0$ so we may set $s=1$. When $d=3, F\left(2,1 ; \frac{1}{2} ; v^{\prime}\right)=0$ for $v^{\prime}=-0.6349132$ and so $v(0) \sim 0.2116377 N$. For general $d$ we may invert (A.4) giving

$$
v^{\prime}(\rho)=\frac{(4-d)(d-2)}{4 N}\left(\rho-\rho_{0}\right)+\mathrm{O}\left(\left(\rho-\rho_{0}\right)^{2}\right), \quad \rho_{0}=\frac{N}{d-2} .
$$

In integrals obtained from the scalar product (3.20) we then have

$$
\rho^{\frac{1}{2} N} e^{-\frac{1}{2}(d-2) \rho-2 v(\rho)} \approx C e^{-\frac{d-2}{2 N}\left(\rho-\rho_{0}\right)^{2}},
$$

for some constant $C$, and so the dominant contribution for large $N$ arises for $\rho \approx \rho_{0}$ where we may use (A.5). Hence

$$
\frac{\left\langle\hat{f}_{1, \phi}, v^{\prime 2}\right\rangle_{1}}{\left\langle\hat{f}_{1, \phi}, 1\right\rangle_{1}} \approx \frac{(4-d)^{2}(d-2)}{16 N} .
$$

Furthermore in (3.28) for $\rho \approx \rho_{0}$

$$
\rho\left(\frac{1}{2}(2-\eta)-v^{\prime}(\rho)-2 \rho v^{\prime \prime}(\rho)\right) \approx \frac{1}{2} N,
$$

so that (3.28) gives

$$
\frac{\left\langle\hat{f}_{1, \phi}, y\right\rangle_{1}}{\left\langle\hat{f}_{1, \phi}, 1\right\rangle_{1}} \approx \frac{d(d-2)(4-d)^{2}}{8(d+2) N} .
$$

Hence from (3.26) the leading large $N$ result for $\eta$ in this analysis is determined to be

$$
\eta=\frac{3 d(d-2)(4-d)^{2}}{8(d+2) N}+\mathrm{O}\left(N^{-2}\right) .
$$

The exact leading order large $N$ result $\eta \sim 2(4-d) \Gamma(d-1) \sin \pi \frac{1}{2}(d-2) /\left(\pi d \Gamma\left(\frac{1}{2} d\right)^{2} N\right)$ is numerically close to (A.10), coinciding as $d \rightarrow 4$. 


\section{B Perturbative Calculations}

We here outline how the perturbative results (6.12) and (6.14) are obtained following the background field methods, with a background field $\varphi$ and dimensional regularisation, used in [21]. To obtain (6.14) we consider vacuum graphs which are $\mathrm{O}(V G)$

$$
\begin{aligned}
W_{1}=\sum_{r \geq 2} & \frac{1}{2 r !} \int \mathrm{d}^{d} x_{1} \mathrm{~d}^{d} x_{2}\left\{G_{i j, i_{1} \ldots i_{r}}\left(\varphi_{1}\right) \partial^{\mu} \varphi_{1 i} \partial_{\mu} \varphi_{1 j} G_{0}\left(x_{12}\right)^{r}\right. \\
& +2 r G_{i i_{1}, i_{2} \ldots i_{r}}\left(\varphi_{1}\right) \partial^{\mu} \varphi_{1 i} \partial_{\mu} G_{0}\left(x_{12}\right) G_{0}\left(x_{12}\right)^{r-1} \\
& \left.+r(r-1) G_{i_{1} i_{2}, i_{3} \ldots i_{r}}\left(\varphi_{1}\right) \partial^{\mu} G_{0}\left(x_{12}\right) \partial_{\mu} G_{0}\left(x_{12}\right) G_{0}\left(x_{12}\right)^{r-2}\right\} V_{i_{1} \ldots i_{r}}\left(\varphi_{2}\right),
\end{aligned}
$$

for $\varphi_{1 i}=\varphi_{i}\left(x_{1}\right)$ etc., and $G_{0}(x)$ the basic $d$-dimensional propagator $-\partial^{2} G_{0}(x)=\delta^{d}(x)$. Using, with $d$ as in (4.1) and $a_{n}$ as in (5.14),

$$
\begin{aligned}
& G_{0}(x)^{n} \sim \frac{2}{\varepsilon} \frac{n ! a_{n}}{n-1} \delta^{d}(x), \quad \partial_{\mu} G_{0}(x) G_{0}(x)^{n-1} \sim \frac{2}{\varepsilon} \frac{(n-1) ! a_{n}}{n-1} \partial_{\mu} \delta^{d}(x), \\
& \partial^{\mu} G_{0}(x) \partial_{\mu} G_{0}(x) G_{0}(x)^{n-2} \sim \frac{2}{\varepsilon} \frac{(n-2) ! a_{n}}{n-1} \partial^{2} \delta^{d}(x) .
\end{aligned}
$$

Consequently the necessary counterterm to cancel the $\varepsilon$-pole in (B.1) is given by

$$
\begin{gathered}
\mathcal{L}_{1 \text { c.t. }}(\varphi)=\frac{1}{\varepsilon} \frac{a_{n}}{n-1} \partial^{\mu} \varphi_{i} \partial_{\mu} \varphi_{j}\left(V_{i_{1} \ldots i_{n}}(\varphi) G_{i j, i_{1} \ldots i_{n}}(\varphi)+2 V_{i_{1} \ldots i_{n} i}(\varphi) G_{j i_{1}, i_{2} \ldots i_{n}}(\varphi)\right. \\
\left.-V_{i_{1} \ldots i_{n} i}(\varphi) G_{i_{1} i_{2}, j i_{3} \ldots i_{n}}(\varphi)\right),
\end{gathered}
$$

which directly gives (6.14).

Correspondingly to obtain (6.12) we consider vacuum graphs which are $\mathrm{O}\left(V^{2} G\right)$

$$
\begin{gathered}
W_{2}=-\frac{1}{2} \sum_{\substack{r, s \geq 0 \\
t \geq 1}} \frac{1}{r ! s ! t !} \int \mathrm{d}^{d} x_{1} \mathrm{~d}^{d} x_{2} \mathrm{~d}^{d} x_{3} G_{i j, i_{1} \ldots i_{r} j_{1} \ldots j_{s}}\left(\varphi_{1}\right) V_{i i_{1} \ldots i_{r} k_{1} \ldots k_{t}}\left(\varphi_{2}\right) V_{j j_{1} \ldots j_{s} k_{1} \ldots k_{t}}\left(\varphi_{3}\right) \\
\quad \times \partial^{\mu} G_{0}\left(x_{12}\right) G_{0}\left(x_{12}\right)^{r} \partial_{\mu} G_{0}\left(x_{13}\right) G_{0}\left(x_{13}\right)^{s} G_{0}\left(x_{23}\right)^{t}
\end{gathered}
$$

By considering the pole in $\left(x_{12}^{2}\right)^{-\lambda_{3}}\left(x_{13}^{2}\right)^{-\lambda_{2}}\left(x_{23}^{2}\right)^{-\lambda_{1}}$ when $\lambda_{1}+\lambda_{2}+\lambda_{3}=d$, assuming $\lambda_{1}, \lambda_{2}, \lambda_{3}<\frac{1}{2} d$ so that there are no sub-divergencies, we find, for $\hat{K}_{r s t}$ as in (6.13),

$$
\begin{aligned}
\partial^{\mu} G_{0}\left(x_{12}\right) G_{0}\left(x_{12}\right)^{r} \partial_{\mu} G_{0}\left(x_{13}\right) & \left.G_{0}\left(x_{13}\right)^{s} G_{0}\left(x_{23}\right)^{t}\right|_{r+s+t=n-1} \\
& \sim \frac{2}{\varepsilon} \frac{n ! a_{n}}{n-1} \frac{\hat{K}_{r+1 s+1 t}}{(r+1)(s+1)} \delta^{d}\left(x_{12}\right) \delta^{d}\left(x_{13}\right), \\
\partial^{\mu} G_{0}\left(x_{12}\right) \partial_{\mu} G_{0}\left(x_{12}\right) G_{0}\left(x_{12}\right)^{r} & \left.G_{0}\left(x_{13}\right)^{s} G_{0}\left(x_{23}\right)^{t}\right|_{r+s+t=n-1} \\
& \sim-\frac{2}{\varepsilon} \frac{n ! a_{n}}{n-1} \frac{\hat{K}_{r+2 s t}}{(r+2)(r+1)} \delta^{d}\left(x_{12}\right) \delta^{d}\left(x_{13}\right) .
\end{aligned}
$$


Hence (B.4) requires the counterterm

$$
\begin{aligned}
\mathcal{L}_{2 \text { c.t. }}(\varphi)=\frac{1}{\varepsilon} \frac{a_{n}}{n-1} & \left(-\sum_{\substack{r, s, t \geq 1 \\
r+s+t=n+1}} \frac{n !}{r ! s ! t !} \hat{K}_{r s t} V_{i_{1} \ldots i_{r} k_{1} \ldots k_{t}}(\varphi) V_{j_{1} \ldots j_{s} k_{1} \ldots k_{t}}(\varphi) G_{i_{1} j_{1}, i_{2} \ldots i_{r} j_{2} \ldots j_{s}}(\varphi)\right. \\
& \left.+\sum_{\substack{r \geq 2, s, t \geq 1 \\
r+s+t=n+1}} \frac{n !}{r ! s ! t !} \hat{K}_{r s t} V_{i_{1} \ldots i_{r} k_{1} \ldots k_{t}}(\varphi) V_{j_{1} \ldots j_{s} k_{1} \ldots k_{t}}(\varphi) G_{i_{1} i_{2}, i_{3} \ldots i_{r} j_{1} \ldots j_{s}}(\varphi)\right),
\end{aligned}
$$

which leads to $(6.12)$.

A consistency check may be obtained by considering (B.6) when $G_{i j}$ is a constant. The result is then $\mathcal{L}_{2 \text { c.t. }}(\varphi)=-\frac{1}{\varepsilon} \frac{n a_{n}}{n-1} V_{i i_{1} \ldots i_{n-1}}(\varphi) V_{j i_{1} \ldots i_{n-1}}(\varphi) G_{i j}$. This may also be obtained directly from lowest order counterterm for $V$ using that the kinetic term in $\mathcal{L}$ defines a metric $\delta_{i j}+G_{i j}$. A similar argument is sufficient to obtain the last line of (6.15).

\section{Analysis with Dimensional Regularisation}

Using dimensional regularisation with minimal subtraction for a theory defined by (5.1) and (6.1) the diffeomorphism invariance under (6.6) extends to the regularised theory and we demonstrate here the consequences for the perturbative $\beta$-functions. The bare lagrangian, including all counterterms involving poles in $\varepsilon$ necessary for finiteness to first order in $F, G_{i j}$, has the general form

$$
\mathcal{L}_{0}=\mu^{-\varepsilon}\left(\frac{1}{2} \partial^{\mu} \phi \cdot Z \partial_{\mu} \phi+\mathcal{V}(\phi)+\mathcal{F}(\phi)+\mathcal{G}_{i j}(\phi) \frac{1}{2} \partial^{\mu} \phi_{i} \partial_{\mu} \phi_{j}\right),
$$

where $\mu$ is the regularisation mass scale and $\mathcal{F}, \mathcal{G}_{i j}$ are linear in $F, G_{k l}$ and $Z_{i j}=Z_{j i}$ depends only on $g_{i_{1} \ldots i_{2 n}}$. The bare couplings and field $\phi_{0}$ are defined so as to absorb all dependence on $\mu$. For the standard renormalisable theory, when $\mathcal{F}, \mathcal{G}_{i j}$ are zero and $\mathcal{V}(\phi)$ is just a polynomial of degree $2 n$, the $\beta$-functions and $\phi$-anomalous dimension are defined through

$$
\begin{aligned}
& \left(-\varepsilon-\left(\hat{\gamma}_{\phi} \phi\right) \cdot \frac{\partial}{\partial \phi}+\hat{\beta}_{V} \cdot \frac{\partial}{\partial V}\right) \mathcal{V}(\phi)=0, \\
& \left(-\varepsilon+\hat{\beta}_{V} \cdot \frac{\partial}{\partial V}\right) Z_{i j}=\gamma_{\phi, i k} Z_{k j}+\gamma_{\phi, j k} Z_{k i},
\end{aligned}
$$

where in terms of the $\beta$-functions considered earlier

$$
\hat{\beta}_{V}(\phi)=-\frac{1}{2} \varepsilon \phi \cdot \partial V(\phi)+\varepsilon V(\phi)+\beta_{V}(\phi), \quad \hat{\gamma}_{\phi, i j}=-\frac{1}{2} \varepsilon \delta_{i j}+\gamma_{\phi, i j} .
$$

For the extended theory, keeping only contributions to first order in $F, G_{i j}$, we also have

$$
\begin{aligned}
& \left(-\varepsilon-\left(\hat{\gamma}_{\phi} \phi\right) \cdot \frac{\partial}{\partial \phi}+\hat{\mathcal{D}}_{\beta}\right) \mathcal{F}(\phi)=0, \\
& \left(-\varepsilon-\left(\hat{\gamma}_{\phi} \phi\right) \cdot \frac{\partial}{\partial \phi}+\hat{\mathcal{D}}_{\beta}\right) \mathcal{G}_{i j}(\phi)=\gamma_{\phi, i k} \mathcal{G}_{k j}(\phi)+\gamma_{\phi, j k} \mathcal{G}_{k i}(\phi),
\end{aligned}
$$


for

$$
\hat{\mathcal{D}}_{\beta}=\hat{\beta}_{V} \cdot \frac{\partial}{\partial V}+\hat{\beta}_{F} \cdot \frac{\partial}{\partial F}+\hat{\beta}_{G, k l} \cdot \frac{\partial}{\partial G_{k l}} .
$$

Here $\hat{\beta}_{F}$ is related to $\beta_{F}$ just as $\hat{\beta}_{V}$ is to $\beta_{V}$ in (C.3) and $\hat{\beta}_{G, i j}(\phi)=-\frac{1}{2} \varepsilon \phi \cdot \partial G_{i j}(\phi)+\beta_{G, i j}(\phi)$.

For transformations as in (6.6) invariance of the regularised theory requires

$$
\begin{aligned}
\left.\mathcal{F}(\phi)\right|_{F=v \cdot \partial V, G_{i j}=\partial_{i} v_{j}+\partial_{j} v_{i}} & =\mathcal{D}_{v} \mathcal{F}(\phi)=\tilde{v}(\phi) \cdot \partial \mathcal{V}(\phi) \\
\left.\mathcal{G}_{i j}(\phi)\right|_{F=v \cdot \partial V, G_{k l}=\partial_{k} v_{l}+\partial_{l} v_{k}} & =\mathcal{D}_{v} \mathcal{G}_{i j}(\phi)=\partial_{i} \tilde{v}_{k}(\phi) Z_{k j}+\partial_{j} \tilde{v}_{k}(\phi) Z_{k i}
\end{aligned}
$$

for

$$
\mathcal{D}_{v}=(v \cdot \partial V) \cdot \frac{\partial}{\partial F}+\left(\partial_{k} v_{l}+\partial_{l} v_{k}\right) \cdot \frac{\partial}{\partial G_{k l}} .
$$

In (C.6a),(C.6b) $\tilde{v}_{i}(\phi)=v_{i}(\phi)+\ldots$ where we may have higher order terms involving poles in $\varepsilon$.

The crucial constraints arise from the consistency conditions between (C.4a), (C.4b) and (C.6a),(C.6b) giving

$$
\begin{aligned}
{\left[\mathcal{D}_{v}, \hat{\mathcal{D}}_{\beta}\right] \mathcal{F}(\phi) } & =\tilde{u}(\phi) \cdot \partial \mathcal{V}(\phi) \\
{\left[\mathcal{D}_{v}, \hat{\mathcal{D}}_{\beta}\right] \mathcal{G}_{i j}(\phi) } & =\partial_{i} \tilde{u}_{k}(\phi) Z_{k j}+\partial_{j} \tilde{u}_{k}(\phi) Z_{k i}
\end{aligned}
$$

where

$$
\left[\mathcal{D}_{v}, \hat{\mathcal{D}}_{\beta}\right]=\left(\mathcal{D}_{v} \hat{\beta}_{F}-v \cdot \partial \hat{\beta}_{V}\right) \cdot \frac{\partial}{\partial F}+\left(\mathcal{D}_{v} \hat{\beta}_{G, k l}\right) \cdot \frac{\partial}{\partial G_{k l}}
$$

and

$$
\tilde{u}_{i}(\phi)=\left(\left(\hat{\gamma}_{\phi} \phi\right) \cdot \frac{\partial}{\partial \phi}-\hat{\mathcal{D}}_{\beta}\right) \tilde{v}_{i}(\phi)-\hat{\gamma}_{\phi, i j} \tilde{v}_{j}(\phi)
$$

To relate $\tilde{v}(\phi)$ to $v(\phi)$ we require that all counterterms are determined by $\mathcal{L}_{0}$. To achieve this we assume

$$
\tilde{v}_{i}(\phi)=v_{i} \cdot \frac{\partial}{\partial F} \mathcal{F}(\phi)
$$

Using (C.10) this leads to

$$
\tilde{u}_{i}(\phi)=\left(v_{i} \cdot \frac{\partial}{\partial F} \hat{\beta}_{F}-\varepsilon v_{i}-\hat{\gamma}_{\phi, i j} v_{j}\right) \cdot \frac{\partial}{\partial F} \mathcal{F}(\phi)+\left(v_{i} \cdot \frac{\partial}{\partial F} \hat{\beta}_{G, k l}\right) \cdot \frac{\partial}{\partial G_{k l}} \mathcal{F}(\phi) .
$$

Comparing (C.8a),(C.8b) and (C.9) with (C.6a),(C.6b) and (C.7) requires

$$
\begin{aligned}
\mathcal{D}_{v} \hat{\beta}_{F}(\phi)-v(\phi) \cdot \partial \hat{\beta}_{V}(\phi) & =u(\phi) \cdot \partial V(\phi), \\
\mathcal{D}_{v} \hat{\beta}_{G, i j}(\phi) & =\partial_{i} u_{j}(\phi)+\partial_{j} u_{i}(\phi),
\end{aligned}
$$

for

$$
u_{i}(\phi)=v_{i} \cdot \frac{\partial}{\partial F} \hat{\beta}_{F}(\phi)-\varepsilon v_{i}(\phi)-\hat{\gamma}_{\phi, i j} v_{j}(\phi) .
$$

These results are equivalent to $(6.8 \mathrm{a}),(6.8 \mathrm{~b})$ with $(6.9)$. 


\section{Singularities of Solutions of RG Equation}

The critical requirement for solving the RG equation (3.1) is that it is necessary to fine tune $k=v(0)$ to ensure there are no singularities for any real positive $\rho$. Nevertheless there are necessarily singularities elsewhere in the complex plane. As shown in [26] it is of interest to determine the location of such singularities so as to allow the use of conformal mapping techniques. The structure of the differential equation determines that the singularities are simple poles of the form

$$
v^{\prime}(\rho) \sim \frac{1}{\rho_{0} e^{i \alpha \pi}-\rho},
$$

where reality of the equation ensures that $\pm \alpha$ must both give singularities unless $\alpha=1$. We restrict then $0<\alpha \leq 1$. The singularities are determined by numerically integrating along lines of constant argument, for $v(0)=k$ and matching with (D.1).

When $d=3$ the only singularities that are found within the radius in which the numerical solution is valid are on the negative real axis, $\alpha=1$. Choosing $\eta=0$ the values of $k$ for suitable $N$ are given in Table 3 and the position of the closest singularity to the origin is given in Table 9.

\begin{tabular}{|r|c|}
\hline$N$ & $\rho_{0}$ \\
\hline 1 & 2.862 \\
2 & 2.836 \\
3 & 2.871 \\
4 & 2.954 \\
10 & 3.871 \\
\hline
\end{tabular}

Table 9: Values of $\rho_{0}$ for $N=1,2,3,4,10$

We have also considered $d=\frac{5}{2}$ since there are then two solutions of physical interest with the additional solution arising for $d<3$ and representing the tricritical fixed point.

For the solution corresponding to the standard Wilson-Fisher fixed point the singularities are again just on the negative real axis. The results are in Table 10. For the tricritical

\begin{tabular}{|r|c|c|}
\hline$N$ & $k$ & $\rho_{0}$ \\
\hline 1 & 0.252995579 & 1.349 \\
2 & 0.800566594 & 1.116 \\
3 & 1.587372474 & 1.014 \\
4 & 2.451092846 & 1.002 \\
10 & 7.725892940 & 1.275 \\
\hline
\end{tabular}

Table 10: Values of $\rho_{0}$ and $k$ for $N=1,2,3,4,10$

case there are genuine complex singularities, results are given in Table 11. An illustrative numerical solution for $v^{\prime}(\rho)$ compared with the pure pole term in (D.1) is shown in Figure 3. 


\begin{tabular}{|r|c|c|c|}
\hline$N$ & $k$ & $\rho_{0}$ & $\alpha$ \\
\hline 1 & -0.043027023 & 6.3636 & 0.5150 \\
2 & -0.132093526 & 6.4736 & 0.4766 \\
3 & -0.279532538 & 6.5380 & 0.4383 \\
4 & -0.499803930 & 6.5544 & 0.3993 \\
\hline
\end{tabular}

Table 11: Tricritical values of $k, \rho_{0}$ and $\alpha$ for $N=1,2,3,4$

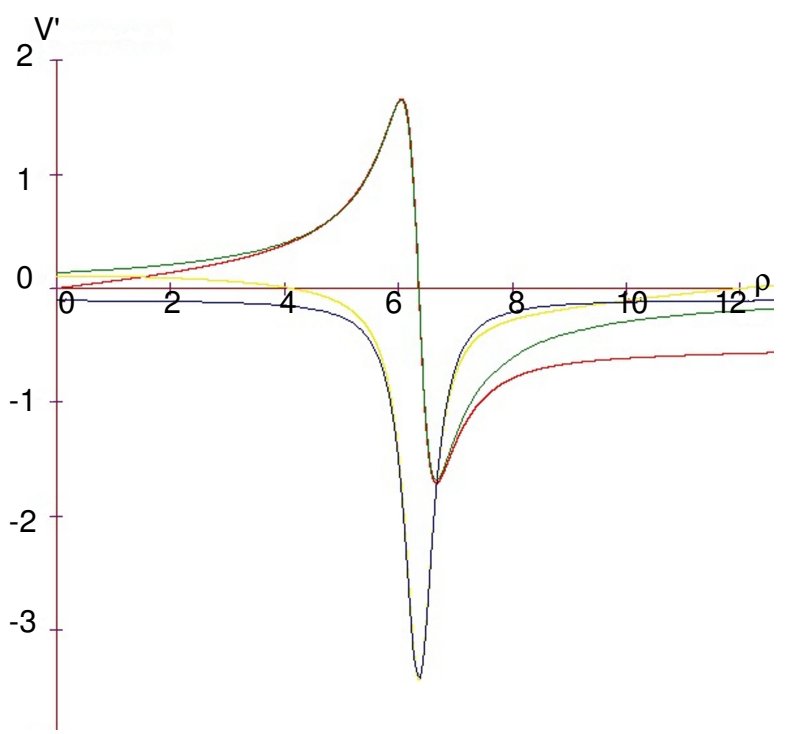

Figure 3: $N=1$ tricritical solution for $v^{\prime}(i \rho)$, red and yellow lines are the real and imaginary parts, the green and blue lines correspond to a pure pole as in (D.1) with $\rho_{0}$ from Table 11

\section{References}

[1] K.G. Wilson, "Renormalization Group and Critical Phenomena. 1. Renormalization Group and the Kadanoff Scaling Picture," Phys. Rev. B4 (1971) 3174.

[2] F.J. Wegner and A. Houghton, "Renormalization Group Equation for Critical Phenomena," Phys. Rev. A8 (1972) 401.

[3] K.G. Wilson and J.B. Kogut, "The Renormalization Group and the Epsilon Expansion," Phys. Rept. 12 (1974) 75.

[4] J. Polchinski, "Renormalization and Effective Lagrangians," Nucl. Phys. B231 (1984) 269.

[5] C. Bagnuls and C. Bervillier, "Exact Renormalization Group Equations. An Introductory Review," Phys. Rep. 348 (2001) 91, hep-th/0002034.

[6] J. Berges, N. Tetradis and C. Wetterich, "Nonperturbative renormalization flow in quantum field theory and statistical physics," Phys. Rept. 363 (2002) 223, hep-ph/0005122.

[7] J.M. Pawlowski, "Aspects of the Functional Renormalization Group," Ann. Phys. 322 (2007) 2831, hep-th/0512261.

[8] B. Delamotte, "An introduction to the nonperturbative renormalization group," condmat/0702365. 
[9] D. F. Litim, "Optimised renormalisation group flows," Phys. Rev. D64 (2001) 10507, hepth/0103195.

[10] J. Distler, "ERGE," http://golem.ph.utexas.edu/\%7Edistler/blog/archives/001609.html.

[11] G.R. Golner, "Nonperturbative Renormalization Group Calculations for Continuum Spin Systems," Phys. Rev. B33 (1986) 7863.

[12] T.R. Morris, "Derivative Expansion of the Exact Renormalization Group," Phys. Lett. B329 (1994) 241, hep-ph/9403340.

[13] R.D. Ball, P.E. Haagensen, J.I. Latorre and E. Moreno, "Scheme Independence and the Exact Renormalization Group," Phys. Lett. B347 (1995) 80, hep-th/9411122.

[14] T.R. Morris, "Properties of derivative expansion approximations to the renormalization group," Int. J. Mod. Phys. B12 (1998) 1343, hep-th/9610012.

[15] J. Comellas, "Polchinski equation, reparameterization invariance and the derivative expansion," Nucl. Phys. B509 (1999) 662, hep-th/9705129.

[16] T.R. Morris, "Three dimensional massive scalar field theory and the derivative expansion of the renormalization group," Nucl. Phys. B495 (1997) 477, hep-th/9612117;

T.R. Morris and M.D. Turner, "Derivative expansion of the renormalization group in $\mathrm{O}(\mathrm{N})$ scalar field theory," Nucl. Phys. B509 (1998) 637, hep-th/9704202.

[17] C. Bervillier, "The Wilson-Polchinski Exact Renormalization Group Equation," Phys. Lett. A332 (2004) 93, hep-th/0405025;

"Wilson-Polchinski exact renormalization group equation for $\mathrm{O}(\mathrm{N})$ systems: Leading and nextto-leading orders in the derivative expansion," J. Phys. Cond. Matt. 17 (2005) S1929, hepth/0501087.

[18] L. Canet, B. Delamotte, D. Mouhanna and J. Vidal, "Optimization of the derivative expansion in the nonperturbative renormalization group," Phys. Rev. D67 (2003) 065004,hep-th0211055; "Nonperturbative renormalization group approach to the Ising model: a derivative expansion at order $\partial^{4}, "$ Phys. Rev. B68 (2003) 064421, hep-th/0302227.

[19] T.L. Bell and K.G. Wilson, "Finite-lattice approximations to renormalization groups," Phys. Rev. B11 (1975) 3431.

[20] T.R. Morris, "Elements of the continuous renormalization group," Prog. Theor. Phys. Suppl. 131 (1998) 395, hep-th/9802039.

[21] J. O'Dwyer and H. Osborn, "Epsilon Expansion for Multicritical Fixed Points and Exact Renormalisation Group Equations", Ann. Phys. 323 (2008) 1859, arXiv:0708.2697.

[22] G. Felder, "Renormalization Group in the Local Potential Approximation," Comm. Math. Phys. 111 (1987) 101.

[23] P.C. Lima, "Renormalization Fixed Points in the Local Potential Approximation for $d \geq 3$," Comm. Math. Phys. 170 (1995) 529.

[24] G.R. Golner and E.K. Riedel, " $\epsilon$-Expansion Solution of Wilson's Exact Renormalization-Group Equation," Phys. Rev. Letters 34 (1975) 171;

P. Shukla and M.S. Green, "Universality of the Exponent $\eta$ to Order $\epsilon^{2}$ for a Class of Renormalization Groups," Phys. Rev. Letters 34 (1975) 436;

J. Rudnick, "Evaluation of $\eta$ in Wilson's Incomplete-Integration Method: Independence of Cutoff Parameters to Order $\epsilon^{2}$," Phys. Rev. Letters 34 (1975) 438.

[25] C. Bervillier, A. Jütter and D.F. Litim, "High-accuracy scaling exponents in the local potential approximation," Nucl. Phys. B783 (2007) 213, hep-th/0701172. 
[26] C. Bervillier, B. Boisseau and H. Giacomini, "Analytic approximation schemes for solving exact renormalization group equations. II Conformal mappings," Nucl. Phys. B801 (2008) 296, arXiv:0802.2970.

[27] J. Comellas and A. Travesset, " $O(N)$ models within the local potential approximation," Nucl. Phys. B498 (1997) 539, hep-th/9701028.

[28] D.F. Litim, "Universality and the Renormalisation Group," JHEP, 0507 (2005) 005, hepth/0503096.

[29] H. Kleinert and V. Schulte-Frohlinde, "Critical Properties of $\phi^{4}$-Theories," World Scientific, Singapore (2001).

[30] J.F. Nicoll, T.S. Chang and H.E. Stanley, "Approximate Renormalization Group Based on the Wegner-Houghton Differential Generator," Phys. Rev. Lett. 33 (1974) 540, (E) 1525; G.F. Tuthill, J.F. Nicoll and H.E. Stanley, "Renormalization-group calculation of the criticalpoint exponent $\eta$ for a critical point of arbitrary order," Phys. Rev. B11 (1975) 4579.

[31] F.J. Wegner in "Phase Transitions and Critical Phenomena, Vol. 6," ed. C. Domb and M.S. Green, Academic Press, New York (1976).

[32] I. Jack and H. Osborn, "General Background Field Calculations with Fermion Fields," Nucl. Phys. B249 (1985) 472.

[33] H. Osborn, "Renormalisation and composite operators in non-linear $\sigma$ models," Nucl. Phys. B294 (1987) 595.

[34] P.S. Howe, G. Papadopoulos and K.S. Stelle, "The background field method and the non-linear $\sigma$-model," Nucl. Phys. B296 (1988) 26.

[35] N. Tetradis and C. Wetterich, "Critical Exponents from the Effective Average Action," Nucl. Phys. B422 (1994) 541, hep-ph/9308214.

[36] J.I. Latorre and T. R. Morris, "Exact scheme independence," JHEP 0011 (2000) 004, hepth/0008123.

[37] Yu.A. Kubyshin, R Neves and R. Potting, "Solutions of the Polchinski ERG equation in the $O(N)$ scalar model," Int. J. Mod. Phys. A17 (2002) 4871, hep-th/0202199. 